\title{
Lab-on-Fiber Technology: a New Avenue for Optical Nanosensors
}

\author{
Marco CONSALES, Marco PISCO, and Andrea CUSANO*
}

\author{
Optoelectronic Division - Department of Engineering, University of Sannio, C.so Garibaldi 107, 82100, Benevento, Italy \\ *Corresponding author: Andrea CUSANO_E-mail: a.cusano@unisannio.it
}

\begin{abstract}
The "lab-on-fiber" concept envisions novel and highly functionalized technological platforms completely integrated in a single optical fiber that would allow the development of advanced devices, components and sub-systems to be incorporated in modern optical systems for communication and sensing applications. The realization of integrated optical fiber devices requires that several structures and materials at nano- and micro-scale are constructed, embedded and connected all together to provide the necessary physical connections and light-matter interactions. This paper reviews the strategies, the main achievements and related devices in the lab-on-fiber roadmap discussing perspectives and challenges that lie ahead.
\end{abstract}

Keywords: Lab-on-fiber, all-in-fiber devices, optical fiber sensors and devices, microstructured fiber Bragg gratings, microstructured optical fibers, multimaterial and multifunctional fibers

Citation: Marco CONSALES, Marco PISCO, and Andrea CUSANO, "Lab-on-Fiber Technology: a New Avenue for Optical Nanosensors," Photonic Sensors, DOI: 10.1007/s13320-012-0095-y.

\section{Introduction}

Ever since their discovery, optical fibers have literally revolutionized the telecommunications industry by providing higher performances, more reliable telecommunications links with ever decreasing bandwidth cost. A beneficial side effect of this revolution is the high volume production of optoelectronic and fiber optic components and the diffusion worldwide of a glassy information superhighway. In parallel with these developments, the fiber optic sensor technology also has been a major user of the technology associated with the optoelectronic and fiber optic communication industry.

It is remarkable that in the last decades, during which the optical fiber has driven the communication revolution, the fiber optic has been conceived mainly as a communication medium. This is probably the reason the fiber optic industry focused its efforts on a small set of materials and structures able to provide light guidance in the fiber core through total internal reflection in the transparency range of silica glass. Also the existing optical fiber devices and components (e.g. gratings, polarizing filters, isolators) are basically constituted by silica glass, and their functionalities are related to the silica glass properties (and the induced variations). In the development of photonic systems, either for the communication or for sensing applications, several out-of-fiber optical components (e.g. light sources, modulators, photo-detectors) are currently employed. A significant technological breakthrough would therefore be the integration and

Received: 22 August 2012 / Revised version: 5 September 2012

(C) The Author(s) 2012. This article is published with open access at Springerlink.com 
development of these components and devices "all-in-fiber". Moreover, the integration of advanced functional materials at micro- and nano-scale, exhibiting the more disparate properties, combined with suitable transduction mechanisms, is the key for the development of highly integrated and multifunctional technological platforms completely realized in a single optical fiber. This achievement would be the cornerstone of a new photonics technological revolution that would lead to the definition of a novel generation of micro and nanophotonic devices all-in-fiber.

The optical fibers are well suited to support this revolution also in virtue of the dynamicity and versatility offered by this technology which, in the last years, has shown further developments in producing specialty fibers. Indeed, a large set of specialty optical fibers have been proposed, ranging from new classes of optical fibers such as microstructured optical fibers (MOFs) $[1,2]$ and plastic optical fibers (POFs) [3, 4], to specific fibers such as double core optical fibers, D-shaped fibers and many others constructed for specific applications.

The optical fiber technology thus constitutes a valuable platform that, combined with the new concept of lab-on-fiber, would enable the implementation of sophisticated autonomous multifunction sensing and actuating systems, all integrated in optical fibers, with unique advantages in terms of miniaturization, light weight, cost effectiveness, robustness and power consumption. Multifunctional labs, able to exchange and manipulate information or to fuse sensorial data, could be realized in a single optical fiber providing auto-diagnostics features as well as new photonic and electro-optic functionalities useful in many strategic sectors such as optical processing, life science, safety and security.

From this perspective, we refer to the lab-on-fiber technology as the basis for the development of a technological world completely included in a single optical fiber, where several structures and materials at micro- and nano-scale are constructed, embedded and connected all together to provide the necessary physical connections and light-matter interactions, useful to provide a wide range of functionalities and unparalleled performances [5].

In the last decade, many research groups focused their efforts on the design and the development of all fiber integrated devices starting with the assessment of the technological steps for their fabrication [1, 6-16]. Two main lines can be traced in the lab-on-fiber roadmap: the first one relies on the local micro- and nano-structuring of optical fibers in order to increase light matter interaction providing the basis for functional material integration. The second one is, instead, mainly directed to find suitable deposition techniques for the integration of such materials at micro- and nano-scale in well defined geometries and shapes.

This paper reviews the strategies, the main achievements and related devices in the lab-on-fiber roadmap discussing perspectives and challenges that lie ahead. In particular, we firstly report the research results concerning the development of several technological platforms implementing the lab-on-fiber concept, carried out in our group at the University of Sannio (Benevento, Italy) as well as in other prestigious research centers. Of course, many other research groups have contributed to this new technological vision, here not reported for brevity. Finally, we focus the attention on new trends involving novel interesting and high potentiality fabrication strategies ranging from advanced multi materials stack and drawing technique up to the use of nanotechnologies, including standard lithographic tools as well as new nano-imprinting approaches.

\section{Labs-on-fiber}

\subsection{Microstructured fiber Bragg gratings}

Microstructured fiber Bragg gratings (MSFBGs) 
represent a first and simple example of device inspired to the lab-on-fiber concept. More specifically, they constitute an emerging class of fiber Bragg gratings (FBGs)-based devices and can be classified in two large categories [6]: the first one relies on FBGs written in MOFs, while the second one involves standard FBGs with localized structural micro-scale defects, created within the hosting fiber, by means of post processing techniques. Another emerging approach (that can be included in the first category) also deserves mention and is based on grating formation within optical fiber nanowires and microwires, that provides a variety of interesting properties, including large evanescent field, flexibility, configurability, high confinement, robustness and compactness [17]. Here, we focus our attention mostly on locally MFBGs, referring to the review paper by Cusano et al. [6] for the analysis and details of the first category.

Basically, a locally MSFBG consists of an FBG with single or multiple localized defects that break the grating periodicity, opening up a plenty of possibilities ranging from photonic bandgap engineering up to surrounding refractive index (SRI) sensitization and functional material integration [6].

The first demonstration of spectral modification induced on ultraviolet (UV) written FBGs was proposed by Canning et al. [18]. Some years later, a photonic band-gap engineering of fiber gratings was demonstrated by thermal post-treatments using localized heating $[19,20]$. An alternative approach for the development of locally MSFBGs was proposed in 2005 by Iadicicco et al. [21], which proposed a partial or complete stripping of the fiber cladding [see in Fig. 1(a)] to produce a local SRI sensitization of the core mode via the evanescent wave (EW) interaction. This, in turn, produces a local change in its effective refractive index (RI) able to form a distributed phase-shift controlled through the optical properties of the surrounding medium [21]. The main spectral effect is the formation of a band-gap inside the stop-band of the grating [see Fig. 1(b)], similarly to the effects observed in phase-shift FBGs [22, 23].

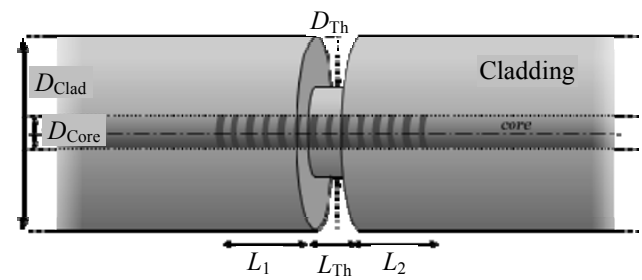

(a)

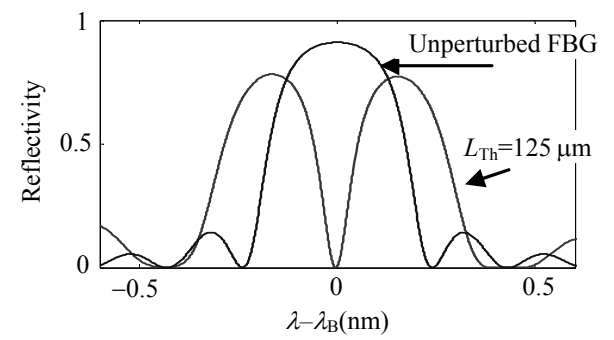

(b)

Fig. 1 Locally MSFBG (a) schematic representation and (b) comparison between the reflected spectra of a $125-\mu \mathrm{m}$ MSFBG and a standard FBG [6].

The spectral position of the defect state inside the stop-band is related to the phase delay introduced by the perturbed region, that is affected by the geometric features of the local defect and the SRI [24]. As the SRI changes, a consequent modification of the effective RI (and thus the phase delay) occurs, leading to a shift in the defect state. A similar behavior occurs when the defect length and the stripping depth are changed. This means that, by a suitable defect design, the spectral tailoring of the final device can be achieved. Moreover, by acting on the SRI, it is possible to actively modulate the defect state, paving the way to the development of all-in-fiber EW sensors and tunable spectral filters [25]. One of the main drawback of this approach is the fiber weakening caused by the local cladding stripping. Particular care is therefore required during the fabrication stage [21]. More advanced lithographic and not-lithographic methods to introduce selective and spatially encoded SRI sensitizations of the guided light were also proposed 
[26-29]. The first one basically includes a masking procedure followed by UV laser micromachining and wet chemical etching [26], while the second one relies on the use of the electrical arc discharge, eventually followed by wet chemical etching [27, 28]. A locally SRI sensitized fiber grating device, involving a micro-slot bypass tunnel across the optical fiber, was also successfully realized by using tightly focused femtosecond laser inscription and chemical etching [30].

The FBG sensitization to the SRI provided by MSFBGs can be exploited for the realization of multiparameter sensing applications and wavelength selective filters. Also, multiple defects can be accomplished along the grating structure, enabling the formation of multiple defect states inside the band-gap where their spectral distribution depends on a proper combination of the geometrical and physical features of all the thinned regions [31].

\subsection{Nano-coated long period gratings}

Long period gratings (LPGs) can also be considered as valuable technological platform to be used for the development of highly functionalized lab-on-fiber devices and components [32]. Thanks to their inherent SRI sensitivity, LPGs only requires proper functional materials integration to open a plenty of new possibilities for both sensing and telecommunications applications, without requiring the local modification of the host fiber. For this reason, they have become increasingly popular devices for the implementation of chemical sensors and biosensors [32].

Bare LPGs are scarcely sensitive in aqueous environment and lack of any chemical selectivity or biological affinity. However, things are very different when a thin layer with sub-wavelength thickness (ranging in hundreds of nanometers) and high refractive index (HRI) (higher than that of the cladding) is deposited onto the fiber surface [33-38] [see Fig. 2(a)]. Indeed, the HRI overlay draws the optical field towards the external medium, extending its EW. As a result, the SRI sensitivity of the device is strongly increased. Moreover, the HRI overlay constitutes a waveguide itself and allows mode propagation depending on its thickness, RI and SRI. For a given material (fixed RI) and overlay thickness, when the SRI is varied in a certain range, the lowest order cladding mode is gradually and completely sucked into the overlay. Its effective refractive index becomes close to the overlay RI leaving a vacancy in the effective refractive index distribution of the cladding modes [see Fig. 2(b)]. At the same time, the effective refractive indices of all the higher order cladding modes shift to recover the previous effective indices distribution. This is reflected through the phase matching condition in the shift of each attenuation band toward the next lower one. In the middle of this modal transition, the attenuation bands can exhibit a sensitivity of thousands of nanometers per refractive index unit (RIU) [39]. The sensitivity characteristics of the coated LPG are drastically modified compared to the bare device [see Fig. 2(c)]. In fact, HRI overlays at nano-scale exhibit a resonant-like SRI sensitivity tailored around the desired SRI by changing the overlay thickness. The SRI sensitivity of LPGs can be optimized at the desired working point (ambient index) through the deposition of a HRI layer by acting on the overlays properties [40].

Several approaches for integrating coatings of sub-wavelength thickness onto the surface of LPGs have been proposed, such as Langmuir-Blodgett (LB) [41], electrostatic self-assembly (ESA) [34] and dip-coating [42, 43] techniques. Recently, a novel approach has also been proposed, based on the alternate deposition of silica nanoparticles (with diameter in the range of $40 \mathrm{~nm}-50 \mathrm{~nm}$ ), using a layer-by-layer (LBL) method [44].

Spectral features shifts as high as thousands of nanometers for a unitary change in the SRI can be easily obtained, and therefore LPGs coated by HRI functional overlays have been successfully exploited for $\mathrm{pH}$, humidity, chemical and biological sensing. 
[45-49]. In particular, coated LPGs can be used as biochemical sensors if the overlay surface is properly functionalized in the way to specifically concentrate target biological molecules (able to produce localized RI variations). In this regard, two approaches have been proposed by our group for the surface functionalization of HRI overlays: in the first one, the hydrophobicity of the HRI overlay (polystyrene) is used to adsorb a protein monolayer of bovine serum albumine subsequently modified to covalently link an antibody [50]; in the second one, a secondary ultra thin layer of poly (methyl methacrylate-co-methacrylic acid) is deposited on a primary polystyrene layer to provide a caroboxyl-containing surface minimizing at the same time its impact on the optical design of the device [51]. For this second approach, we developed an original solvent/non-solvent strategy for the correct formation of the polymer multilayer structure.

Polymeric coated LPGs working in transition mode have also been used as highly sensitive devices for the monitoring of nano-scale phenomena occurring at the polymer/water interface, that have been successfully exploited for cation sensing [52].

\subsection{Evanescent wave LPGs within D-shaped optical fibers}

D-shaped optical fibers, due to their planar side, are particularly suited to optical fiber structuring processes as well as to materials integration. They constitute a very interesting platform as they allow access to light in the core by removal of a thin cladding layer. D-fiber etching was adopted for the development of SRI sensitive gratings by Meltz et al. in 1996 [53]. Successively, researchers at the Brigham Young University demonstrated how to etch an arbitrary length of the D-fiber to remove and replace the core section with another (functional) optical material [54]. The same group proposed, for the first time, a surface relief FBG realized on the flat surface of a D-shaped optical fiber and exploited such device for the realization of various sensor typologies such as high temperature sensors [55], strain sensors [56], and chemical sensors [57].

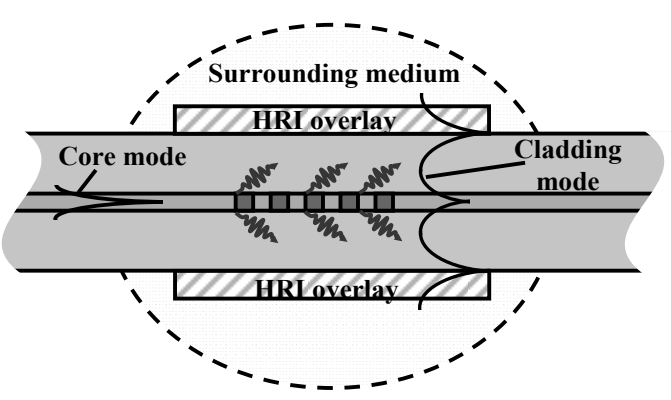

(a)

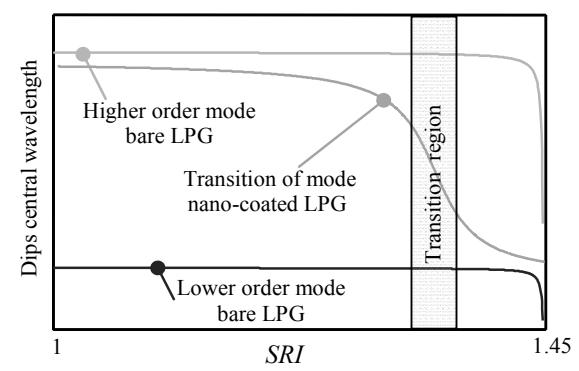

(b)

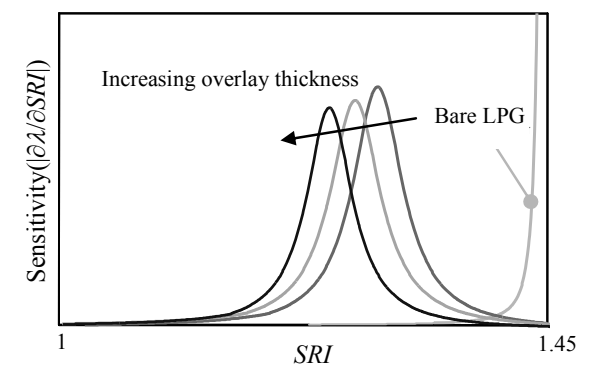

(c)

Fig. 2 LPG coated by a HRI overlay (a) schematic representation, (b) mode transition, and (c) sensitivity characteristic.

Jang et al. also recently proposed an evanescent wave LPFG (EWLPFG) for biosensing applications by using a side-polished fiber in combination with periodically patterned photo-resist using photolithography [58]. A uniform resist layer was spun on a side-polished fiber and successively patterned using a photolithographic process. To investigate the bio-sensing performance of such a device, further functionalization of the side-polished glass fiber substrate were carried out. Even if the 
basic idea is very interesting, the proposed approach shows certain limitations, first of all the restricted overlay types to be adopted (only photo-resist). In addition, the final device has to be properly functionalized for the specific application therefore requiring suitable post-processing stage. Finally, photo-resist cannot be easily functionalized for bio-sensing, severely restricting the versatility of the proposed method.

To overcome this limitation, our group recently proposed a different approach for the realization of an EWLPFG, based on the periodic patterning (via UV laser micromachining) of a polymeric overlay (polystyrene) deposited onto a "sensitized" D-fiber (see Fig. 3) [59]. The use of the D-shaped fiber as the fiber substrate simplifies the realization procedure as it enables to avoid the need to block the fiber in a bulk material for deep side-polishing processes.

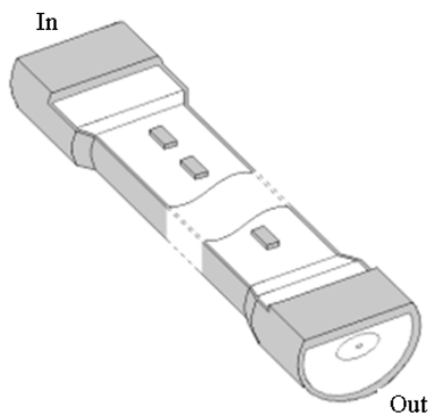

(a)

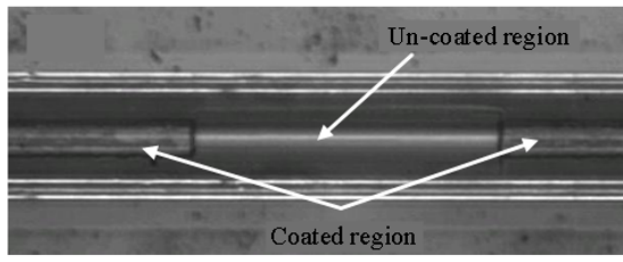

(b)

Fig. 3 EWLPFG realized within a D-shaped optical fiber (a) schematic representation and (b) optical microscope image of the periodically patterned polystyrene overlay [59].

SRI sensitivity around the water RI of about $700 \mathrm{~nm} / \mathrm{RIU}$ were demonstrated, more than one order of magnitude higher than those of the standard LPFG characterized approximately by the same period (SRI sensitivity in water: $8 \mathrm{~nm} / \mathrm{RIU}$ $19 \mathrm{~nm} / \mathrm{RIU}$ ) [60] and a bit smaller than those achieved using the best two LPFG configurations: the HRI coated LPFG ( $>1000 \mathrm{~nm} / \mathrm{RIU})$ [50] and the LPFG written in a micro-structured optical fiber with optimized geometry $(\sim 1500 \mathrm{~nm} / \mathrm{RIU})$ [61]. However, it is worth considering that no optimization of the device parameters were carried out for the proposed EWLPFG, thus meaning that further optimization margin exists for achieving higher SRI sensitivity in water, reducing the gap with the other configurations. Finally, it is important to stress the main advantage of the exploited technique, i.e. its flexibility: materials of different natures could be selected and opportunely deposited onto the flat-surface of the D-fiber in order to realize self-functionalized EWLPFGs suitable for specific applications, while the sensitivity can be tuned by acting directly on the D-fiber during the chemical etching stage.

\subsection{Nano-structured functional materials for lab-on-fiber components}

As previously discussed, the definition of a technological environment for the realization of functional lab-on-fiber devices and components requires also the capability to integrate, pattern and functionalize advanced materials with the specific properties at micro- and nano-scale onto and within optical fibers platforms. Different in-fiber devices have been proposed, based on the integration of the optical fibers with functionalized overlays. In all investigated cases, the basic principle is to take advantage of the changes in the optical properties of the sensitive overlays induced by the chemical interaction with target analytes to produce modulated light signals. Also, a common feature adopted in all proposed schemes relies on the use of sensitive overlays at sub-wavelength scale.

\subsubsection{Carbon nanotubes integration with optical fibers}

Since their discovery, carbon nanotubes (CNTs) have been extensively studied as nano-structured materials for many nanoscience applications due to 
their unique outstanding characteristics. In particular, the distinctive morphology of CNTs (their peculiar hollow structure, nanosized morphology and high surface area) confers them the amazing capability to reversibly adsorb molecules of numerous environmental pollutants undergoing a modulation of their electrical, geometrical and optical properties, such as the resistivity, dielectric constant, thickness. CNTs-based chemical sensors thus offer the possibility of excellent sensitivity, low operating temperature, rapid response time and sensitivities to various kinds of chemicals [62].

The peculiarity of single-walled CNTs (SWCNTs) to change their optical properties due to the adsorption of environmental pollutants was for the first time demonstrated in 2004, when nano-scale CNTs-based overlays were integrated with standard optical fibers for the realization of fiber optic Fabry-Perot (FP) based sensors for environmental monitoring [63, 64]. In that case, thin films of SWCNT bundles were transferred on the fiber facet by means of the molecular engineered LB technique and used as sensitive elements for the development of volatile organic compounds (VOCs) chemo-sensors. Since then, different configurations involving buffered and not buffered [65, 66] SWCNTs overlays, as well as CNTs-based nano-composites layer [67], were successfully integrated on the tip of standard fibers by the LB methods. The realized devices demonstrated excellent sensing capabilities towards VOCs and other pollutants in different environments (air or water) and operating conditions (room temperature or cryogenic temperatures) $[68,69]$. The main achieved results are summarized in Table 1.

The CNTs integration with the optical fiber technology, aiming at the development of all-in-fiber devices, has been the subject of intense research activities also in the field of photonic components. Here, indeed, they received a great deal of interest since 2004, when for the first time CNTs were exploited for the realization of saturable absorbers (SA) [70]. CNTs-based SA demonstrated numerous key advantages compared to other SA such as the small size, ultra-fast recovery time, polarization insensitivity, high optical damage threshold, mechanical and environmental robustness, chemical stability, tunability to operate at wide range of wavelength bands, and compatibility to optical fibers. Using CNT-based SA, the group of Prof. Yamashita, at the University of Tokyo, successfully realized femtosecond fiber pulsed lasers at various wavelengths as well as very short cavity fiber laser having high repetition rates. To this aim, different techniques were exploited for CNTs integration (in the form of a few micrometer-thick layer) either on optical fiber ends or on the flat surface of D-shaped optical fibers, such as the spray method [70], chemical vapor deposition method [71] or novel optical methods based on the use of light [72]. Besides SA, several kinds of CNTs-based non-linear optical devices were also proposed, exploiting their high third-order non linearity. Just to name a few, an all-optical wavelength converter based on the nonlinear polarization rotation as well as a four-wave mixing-based wavelength converter were realized by using CNTs-coated D-shaped optical fibers [73].

Table 1 Main results so far achieved with opto-chemical nanosensors realized by the integration of CNTs onto the end-face of standard optical fibers.

\begin{tabular}{c|c|c|c|c}
\hline \multirow{2}{*}{$\begin{array}{c}\text { Chemical } \\
\text { detection }\end{array}$} & \multicolumn{2}{|c|}{ Room temperature } & \multirow{2}{*}{ Cryogenic } \\
\cline { 2 - 4 } in air & in water & temperatures \\
\hline \multirow{2}{*}{ Target } & $\begin{array}{c}\text { Hydrocarbons, } \\
\mathrm{NO}_{2}\end{array}$ & Alcohols & Toluene & Hydrogen \\
\hline Detection limit & $\sim 300 \mathrm{ppb}$ & $\sim 1 \mathrm{ppm}$ & $<1 \mathrm{ppM}$ & $<1 \%$ \\
\hline Response time & $\sim 10$ minutes & $<1$ minute & $\sim 15$ minutes & $\sim 4$ minutes \\
\hline
\end{tabular}

\subsubsection{Near-field opto-chemical sensors based on $\mathrm{SnO}_{2}$ particle layers}

Metal oxides are widely used sensitive materials for electrical gas sensors in environmental, security 
and industrial applications. In particular, tin dioxide $\left(\mathrm{SnO}_{2}\right)$ was one of the first considered and is still one of the most used material for these applications, thanks to its excellent sensing performance in the presence of small amounts of a wide range of gases $[74,75]$. To exploit the amazing sensing properties of such material also for optical sensing, in 2006, for the first time, our group proposed the integration of $\mathrm{SnO}_{2}$ particle layers onto the end facet of a single mode optical fiber for the development of near-field optical fiber chemo-sensors [76, 77]. To this aim, a proper customization of the simple and low-cost electrostatic spray pyrolysis deposition technique was used, which allowed the tailoring of the structural properties of the $\mathrm{SnO}_{2}$ film such as the crystalline size, thickness, and porosity by simply changing the deposition process parameters (such as the metal chloride concentration, solution volume and substrate temperature). This novel tin dioxide-based near-field sensors exhibited a surprising capabilities of detecting either aromatic hydrocarbons in air or ammonia trace in water, at room temperature, with sensors resolution of the order of a few tens of ppb [78]. In particular, the sensitive layer morphology was demonstrated to be a key aspect for optical sensing because it is able to significantly modify the optical near-field profile emerging from the film surface (see Fig. 4). As a matter of fact, local enhancements of the EW contribution occurs leading to a strong sensitivity to surface effects induced as a consequence of analyte molecule interactions [78, 79]. Indeed, the obtained sensitivities turned out to be an order of magnitude higher than those obtained with the sensors in the standard FP configuration (i.e. with almost flat $\mathrm{SnO}_{2}$ layer on the fiber tip). This demonstrates that the achieved amazing results can be explained by the fact that the interaction between analytes molecules and the sensitive material occurs mainly on the $\mathrm{SnO}_{2}$ surface by means of the optical near-field, with a significant enhancement of the evanescent part of the field.

Recently, our group has also been engaged to investigate the possibility to exploit the fiber optic sensing technology as novel fiber optic humidity sensors to be applied in high-energy physics (HEP) applications, and in particular in experiments actually running at the European Organization for Nuclear Research (CERN).

The technologies currently in use at CERN for RH monitoring are mainly based on polymer capacitive sensing elements with on-chip integrated signal conditioning that needs at least three wires for each sensing point [80]. This means that a complex cabling is required to control the whole volume inside the tracker with enough $\mathrm{RH}$ sensors. In addition, these devices are critically damaged when subject to high radiations doses, being not designed with radiation hardness characteristics. Optical fiber sensors provide many attractive characteristics, including the reduced size and weight, immunity to electromagnetic interference, passive operation (intrinsically safe), water and corrosion resistance. In addition, modern fiber fabrication technologies allow obtaining fibers with low to moderate levels of radiation induced attenuation. In this scenario, near-field fiber optic sensors based on particle layers of tin dioxide have been employed to monitor even low values of relative humidity $\mathrm{RH}$ at low temperatures $\left(20{ }^{\circ} \mathrm{C}\right.$ and $\left.0{ }^{\circ} \mathrm{C}\right)$. The $\mathrm{RH}$ sensing performance of fabricated probes was analyzed during a deep experimental campaign carried out in the laboratories of CERN, in Geneve [81]. Obtained results evidenced that fiber optic sensor responses were in very good agreement with those provided by the commercial polymer-based hygrometers in either static or dynamic measurement, with very high sensitivity and limits of detection less than $0.1 \%$ for the low RH range $(<10 \%)$, thus evidencing the strong potentialities of the fiber technology in light of its future exploitation as robust and valid alternative to currently used polymer-based devices 
for RH monitoring in HEP.

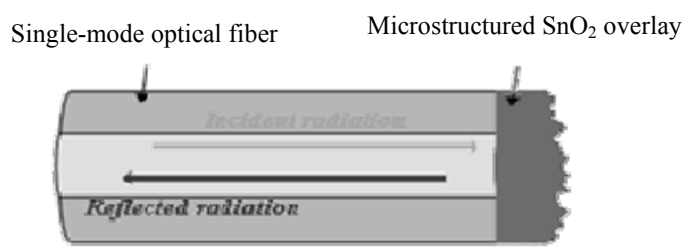

(a)

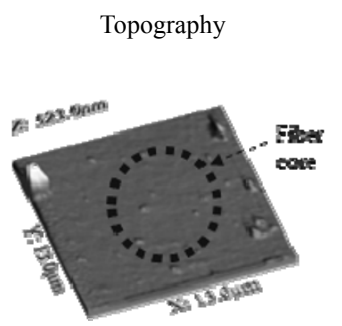

Near-field intensity

Topography

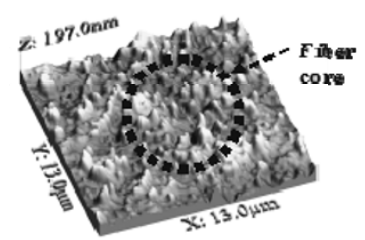

(b)

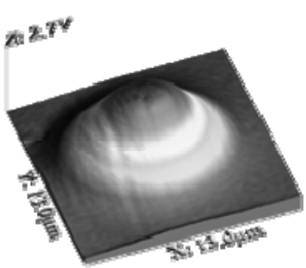

Near-field intensity

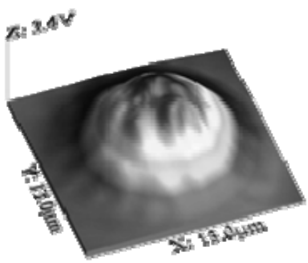

Fig. 4 Near-field opto-chemical sensors based on the particle layers sensing configuration (a) schematic representation and (b) atomic force microscopy images of a flat and micro-structured layers deposited on the fiber tip and corresponding optical near-field collected by the near-field scanning microscope probe in the same region.

\subsection{Functional materials integration in MOFs}

MOFs are highly valuable for the development of advanced all-in-fiber components with specific and often unique characteristics. Due to their peculiar composite structure, MOFs offer a high degree of freedom in their design, whereas the holey structure potentially allows the integration of specific materials able to confer them additional functionalities. So far, a wide range of MOFs have been proposed, and a wide class of devices has already been designed and fabricated [1, 2, 9]. In particular, MOFs constitute a fascinating technological platform for sensing applications. Indeed, they have the potential for dramatically improving the performance of standard fiber optic sensors, as a significant portion of the guided light can be located in the fiber holes in order to maximize the overlap with the material to be sensed.

In particular, a large number of optical fiber refractometers based on FBGs written in MOFs have already been proposed. In 2006, Phan Huy et al. firstly explored the RI sensitivity of FBGs written in one-ring six-hole fibers and two-ring six/twelve-hole fibers [82]. The same group successively presented a uniform FBG photo-written in an MOF with a suspended core fiber, usually called steering-wheel fiber, for further enhancing the RI sensitivity [83].

Optical fiber refractometers find application in chemical and biological sensing, where RI transducers are able to supply information on chemical species as well as biological targets concentrations, but they are also valuable building blocks to be integrated with sensing materials in order to implement highly specific chemical and biological sensors.

Chemical and biological sensing has been demonstrated using absorption spectroscopy in MOFs [84, 85] and captured fluorescence-based sensing in liquid-filled hollow-core MOFs [86, 87], side excited MOFs [88, 89], and double-clad and multi-core (liquid filled) MOF [90]. Both in-fiber excitation and fluorescence recapturing within a liquid-filled solid-core MOF have been also demonstrated [91]. Ritari et al. studied gas sensing in air-guiding photonic bandgap fibers (PBFs) by filling them in turn with hydrogen cyanide and acetylene at low pressure and measuring absorption spectra [92]. Smolka et al. studied the sensing performances of the MOFs for absorption and fluorescence measurements by infiltrating a few nanoliter of a dye solution directly in the holey structure [87-89]. In a wider perspective, the microstructuration of the cladding as well as the presence of the air core, allows the development of new functionalized devices by filling the MOF holes with suitable functional materials [93]. Most of the filling procedures typically involve straightforward 
filling of the holes using a pressure system, whether it is gaseous, liquid, metal or indeed solid powder; some involve reactions within the channel "cells" [94]. Other interesting work involves inserting liquid crystals within structured photonic crystals (PCs) and PBFs to make various components [95-97], as well as metal filling to generate metal assisted mode propagation [98]. Also by manipulating the properties of the in-fiber integrated materials, the guiding features of the MOF itself can be properly changed in order to develop new tunable photonic devices [86, 95, 96, 99].

The integration between MOFs and specific sensing materials can enable the development of novel opto-chemical sensors, exploiting the change in the guiding properties of hollow core optical fibers (HOFs) due to the presence of the sensitive material within the holey structure. In 2006, our group integrated SWCNTs with the HOFs to realize sensing probes basically composed of a piece of HOF, spliced at one end with a standard silica optical fiber and covered and partially filled with SWCNTs at the other termination [100, 101]. The sensing capabilities of the proposed device were demonstrated towards tetrahydrofuran and other VOCs at room temperature.

Selective filling of the cladding holes or of the cores of MOFs is an important step towards the realization of composite structures and can be achieved, whether by plugging or by sealing the holes [86, 102-106]. A simple example based on blocking a larger hole with polymer is the filling of a core of a PBF with one liquid and the cladding with another liquid [107]. Controlled splicing was recently used to report the selective filling of holes with three dyes (selected to be luminescent in the blue, green and red), inserted around the core of a regular structured fiber [108]. The laser dyes after excitation generate white light propagating in the fiber core as blue, green and red light are trapped [94]. The accessibility to the air channels of the PCF has opened up endless possibility for functionalization of the channel surfaces. In particular, there has been a growing interest in imparting the functionality of surface-enhanced Raman scattering (SERS) in MOFs for sensing and detection. For a wide survey on sensors based on MOFs for SESR see [109, 110]. Just to name a few, a special MOF characterized by four big air holes inserted between the solid silica core and the PC cladding holes has been employed for SERS detection [111]. The gold nanoparticles, serving as the SERS substrate, have been either coated on the inner surface of the holes or mixed in the analyte solution infiltrating in the holes via the capillary effect. The SERS signal generated by the lighting probe was collected into the Raman spectrometer to efficiently detect a testing sample of the solution of Rhodamine B. Similarly, SERS characterization with silver nanoparticles deposited on the inner wall of MOFs has also been demonstrated [112]. Yan et al. presented a HOF SERS probe with a layer of gold nanoparticles coated on the inner surface of the air holes at one end of the fiber. HOFs allowed the direct interaction of light excitation with the analyte infiltrated in the core of the optical fiber. In addition, Du's group reported a SERS-full-length active solid core MOF platform with immobilized and discrete silver nanoparticles [113-115]. The sensing probe requires a uniform incorporation of SERS active nanostructures inside the hollow-core along the entire fiber length with a controlled surface coverage density, but in this configuration the entire length of the PCF can be utilized for SERS detection in forward propagating mode by increasing the measured SERS signal due to the increased interaction path [109].

\subsection{Self-assembly of nano-structured materials onto optical fibers}

Self-assembly of nano-structured materials holds promise as a low-cost, high-yield technique with a wide range of scientific and technological applications [116]. Among the various nano-scale 
deposition techniques, the LBL ESA has been often employed to integrate nanomaterials with optical fibers. LBL is able to build up nanometric coatings on a variety of different substrate materials such as ceramics, metals, and polymers of different shapes and forms, including planar substrates, prisms, convex and concave lenses. The deposition procedure for all cases is based on the construction of molecular multilayers by the electrostatic attraction between oppositely charged poly-electrolytes in each monolayer deposited and involves several steps. The versatility of the LBL method for the synthesis of materials enables the application of this technique to the fabrication of different structures on the fiber facet or on the cladding of the optical fiber. Optical fiber sensors based on nano-scale Fabry-Perots, microgratings, tapered ends, biconically tapered fibers, LPGs or PC fiber have made possible the monitoring of temperature, humidity, $\mathrm{pH}$, gases, $\mathrm{VOC}, \mathrm{H}_{2} \mathrm{O}_{2}$, copper or glucose $[49,117,118]$.

\subsection{Surface plasmon resonance optical fiber sensors}

The exploitation of the surface plasmon resonance (SPR) phenomenon for chemical and biological sensing has significantly attracted the attention of the researchers due to the extraordinary sensitivities they are able to provide. Plasmons indeed are exceptionally sensitive reporters for chemical phenomena that influence the RI of the local environment of a probe [119-121]. SPR sensors based on optical fibers are, in turn, particularly attractive for chemical and biological sensing for the intrinsic advantages associated with the use of the fiber optic technology. The challenge in exploiting the SPR phenomena for optical fiber sensors relies on the difficulty in the implementation of a coupling method for surface plasmon excitation in the fiber. In spite of this, several SPR fiber sensor configurations have been proposed in the last decades. Two very recent reviews collects the relevant results obtained in micro- and nano-structured optical fiber sensors, and wide space is reserved to SPR fiber sensors [11, 122]. These include symmetrical structures, such as a simple metal coated optical fiber with and without the cladding layer [123, 124], tapered fiber [125-127], structures based on specialty fibers, such as the D-shaped or H-shaped fiber and one-side metal coated fibers with and without remaining cladding [128-130], and structures with modified fiber tips with flat or angled structures [131, 132]. Each of them may contain either an overlayer (i.e. a layer deposited on the metal laye) or a multilayer structure, which can be used to tune the measurable range for a fiber-optic SPR sensor [131-135]. Many SPR fiber sensor systems have also been developed with structures that have been further modified, via the use of several types of gratings, hetero core fibers, nano-holes etc. [11].

Some of the recently proposed structures include the use of various types of fiber gratings in SPR fiber sensors. Among them, He et al. suggested an SPR fiber sensor adopting cascaded LPGs [136]. Tang et al. also employed an LPG to develop an SPR fiber sensor with self-assembled gold colloids on the grating portion; the sensor was intended for the use in sensing the concentration of a chemical solution and for the label free detection of bimolecular binding at a nano-particle surface [137]. An SPR fiber sensor with an FBG for SPP excitation was described by Nemova and Kashyap [138]. An SPR sensor using an FBG was also proposed recently, in which HOFs were adopted [139]. Other types of gratings have also been investigated, including tilted fiber gratings [140] and metallic Bragg gratings [141]. Many types of SPR sensors based on PCFs have also been proposed [120, 121, 142-145]. As an example, in 2008, Hautakorpi et al. proposed an SPR sensor based on a three-hole microstructured optical fiber with a gold layer 
deposited on the holes.

\section{Towards advanced lab-on-fiber configurations}

Although the works described in the previous sections led to all-in-fiber devices and can therefore be viewed within the lab-on-fiber concept, their main limitations rely on the impossibility to provide a fiber micro- and nano-structuring as well as a functional material definition at the sub-wavelength scale in a controlled manner. This also prevents the exploitation of many novel and intriguing phenomena that are at the forefront of the scientific optical research, involving light manipulation phenomena and excitations of guided resonances in PCs [146, 147] and quasi-crystals [148-151] as well as combined plasmonic and photonic effects in hybrid metallo-dielectric structures [152]. This means that more sophisticated fabrication strategies and technologies need to be used to create the necessary technological scenario for advanced lab-on-fiber components and devices.

\subsection{Multi-material fibers: macro-to-micro approach}

In the past years, a prestigious group at the Massachusetts Institute of Technology (MIT) has been particularly involved in research activities devoted to the integration of conducting, semiconducting, and insulating materials with optical fibers, thus giving rise to specific multimaterial fibers, each of them defined by its own unique geometry and composition. The technological strategy in achieving this goal exploited a preform-based fiber drawing technique consisting in the thermal scaling of a macroscopic multi-material preform [12].

The key to this approach is the identification of materials that can be co-drawn and are capable of maintaining the preform geometry in the fiber and the prevention of axial- and cross-sectional capillary break-up. The following general conditions, highlighted by the same authors, have to be satisfied with the materials used in this process:

(1) At least, one of the fiber materials needs to support the draw stress and yet continuously and controllably deform (thus it should be amorphous in nature and resist devitrification, allowing for fiber drawing with self maintaining structural regularity).

(2) All the materials (vitreous, polymeric or metallic) must flow at a common temperature.

(3) The materials should exhibit good adhesion/wetting in the viscous and solid states without cracking even when subject to rapid thermal cooling.

All these requirements lead inevitably to a reduction in available materials to integrate in all-in-fiber devices by the MIT approach. Specifically, the classes of materials employed to fabricate multimaterial fibers are chalcogenide glasses and polymeric thermoplastics. In producing optoelectronic fiber devices, metals need to be co-drawn with glasses and polymers. In addition, only low-melting-point metals or alloys are suitable for the thermal-drawing process.

Even if the proposed approach suffers from some limitations on the class of materials to be used and on the implementable geometry (necessarily longitudinally invariant), it demonstrated great potentialities. As a matter of fact, several distinct fibers and fiber-based devices were realized by this approach [12, 153-161]. Photodetecting fibers, which behave as photodetectors with sensitivity to visible and infrared light at every point along its entire length, were obtained from a macroscopic preform consisting of a cylindrical semiconductor chalcogenide glass core, contacted by metal conduits encapsulated in a protective cladding [153-156]. Another example of multimaterial fiber is a hollow-core photonic bandgap transmission line surrounded with a thin temperature-sensitive semiconducting glass layer, which is contacted with electrodes to form independent heat-sensing devices [157-159]. An omnidirectional reflecting multilayer 
structure, surrounded with metallic electrodes, has been also employed to allow simultaneous transport of electrons and photons along the fiber [158]. A solid nano-structured fiber consisting of a chalcogenide glass core and a two-dimensional PC cladding has been proposed to generate supercontinuum light at desired wavelengths when the highly nonlinear core is pumped with a laser [159]. Finally, multimaterial piezoelectric fibers have been recently obtained by thermally drawing a structure made of a ferroelectric polymer layer, spatially confined and electrically contacted by internal viscous electrodes and encapsulated in an insulating polymer cladding. The fibers show a piezoelectric response and acoustic transduction from $\mathrm{kHz}$ to $\mathrm{MHz}$ frequencies $[160,161]$.

\subsection{Nano-transferring approach}

Alternatively to the thermal scaling, a promising nanofabrication method has been proposed by an excellent group at the Harvard University [13], enabling nano-scale metallic structures, created with electron-beam lithography (EBL), to be transferred to unconventional substrates (small and/or non-planar) that are difficult to pattern with standard lithographic techniques. The approach is based on the use of a thin sacrificial thiol-ene film that strips and transfers arbitrary nano-scale metallic patterns from one substrate to another, and it was successfully exploited to transfer a variety of gold and silver patterns and features to the facet of an optical fiber. Using this novel transfer technique, a bidirectional fiber based probe was already proposed and demonstrated for in-situ SERS detection [162]. Although the method is said by the authors to be immediately applicable to other metals, such as platinum and palladium, which bind to thiols strongly and to silicon weakly, some limitations occur for a simple extension to other materials for which a proper selection of substrates and design of interfacial chemistry have to be performed. In particular, eligible materials must (1) be able to be patterned on a flat substrate without forming covalent bonds with the substrate and (2) have a surface functionality that allows the material to be stripped. In addition, the adhesive strength of nanoparticles to the fiber facet is too low to endure even some kinds of cleaning processes and too many intermediate and high precision fabrication steps need to be carried out, from the nano-scale pattern fabrication performed on planar substrates to its transfer onto the facets of optical fibers.

The same group at Harvard University recently proposed a further integrated approach for fabricating and transferring patterns of metallic nanostructures to the tips of optical fibers [14]. The process combines the technique of nanoskiving (e.g. the thin sectioning of patterned epoxy nanoposts supporting thin metallic films to produce arrays of metallic nanostructures embedded in thin epoxy slabs) with manual transfer of the slabs to the fibers facet. Specifically, an ultramicrotome equipped with a diamond knife is used to section epoxy nano-scale structures coated with thin metallic films and embedded in a block of epoxy. Sectioning produces thin epoxy slabs, in which the arrays of nanostructures are embedded, that can be physically handled and transferred to the fiber tip.

For the transfer, the floating slab is positioned on the surface of a water bath and is then captured with the tip of the fiber by holding the fiber with tweezers and pressing down manually on the slabs. As the slab is driven under the surface of the water using the tip, it wraps itself around the tip. Withdrawing the tip from the water bath, the slab results irreversibly attached to the tip. Finally, an air plasma is used to etch the epoxy matrices, thus leaving arrays of metallic structures at the nano-scale on the fiber facet. The proposed technique, so far demonstrated to transfer several nanostructure typologies such as gold crescents, rings, high aspect-ratio concentric cylinders, and gratings of 
parallel nanowires, is said by the authors to enable the realization of optical components useful for many applications, including sensing based on localized SPR (LSPRs), label-free detection of extremely dilute chemical and biological analytes using SERS, optical filters diffraction gratings and nose-like chemical sensors. However, it still presents two main limitations: the first is related to the weak adhesion of the structures to the glass surface (that are attached to the fiber through Van der Waals forces); the second is the possible formation of defects that can occur due to the mechanical stresses of sectioning, combined with the intrinsic brittleness of evaporated films and the compressibility of the epoxy matrix (mainly fracture of individual structures and global compression in the direction of cutting), and due to the folding of the slabs during the transfer procedure (formation of crease running across the core of the fiber).

Based on the same transfer technique, in 2009 a highly sensitive RI and temperature monolithic silicon PC fiber tip sensor was also proposed [163]. The PC slab was firstly fabricated by reactive ion etching (RIE) on standard wafers in a silicon foundry, and successively it was released, transferred and bonded to the facet of a single-mode fiber using a micromanipulator and focused ion beam (FIB) tool. The realized PC fiber tip sensors revealed very high sensitivity to RI and temperature and have the attractive feature of returning a spectrally rich signal with independently shifting resonances that can be used to extract multiple physical properties of the measurand and distinguish between them.

Among the transfer methods, it deserve mention also the UV-based nano-imprint and transfer lithography technique proposed by Scheerlinck et al. as a flexible, low-cost and versatile approach for defining sub-micron metal patterns on optical fiber facets [164]. This technique was applied for the fabrication of optical probes for photonic integrated circuits based on a waveguide-to-fiber gold grating coupler.

\subsection{Micro- and nano-technologies directly operating on optical fiber substrates}

An alternative approach to achieve advanced lab on fiber configurations could be the use of microand nano-technologies directly operating on fiber substrates, thus avoiding the critical transferring step. This means that well assessed technologies for both deposition (spin coating, dip coating, sputtering, evaporation, etc..) and sub-wavelength structuring (FIB, EBL, RIE, etc..) have to be adapted to operate on optical fibers taking into account the particular geometry of the substrates.

The management, assessment and conjunction of advanced micro- and nano-machining techniques, enriched by the technological capability to integrate, pattern and functionalize advances materials at micro- and nano-scale onto and within micro- and nano-structured optical fibers can be envisioned as the key aspect to define a technological environment for lab-on-fiber implementation and advanced functional device realization.

Following this technological strategy, valuable advanced fiber optic devices have already been realized in the recent years. In particular, fiber-top cantilevers have been realized directly onto the fiber top by means of nano-machining techniques such as FIB [165] and femtosecond laser micromachining [166]. Similarly, ferrule-top cantilevers have been machined by ps-laser ablation on the top of a millimeter sized ferrule that hosts an optical fiber [167]. They both are easily exploitable in sensing applications. Indeed, light coupled from the opposite end of the fiber allows to remotely detect the tiniest movement of the cantilever. Consequently, any event responsible for that movement can be detected. The vertical displacement of the cantilever can be measured by using standard optical fiber interferometry and starting from this configuration, applications for chemical sensing [168], RI 
measurement [169] and atomic force microscopy [170] have already been reported.

In 2008, Dhawan et al. proposed the use of FIB milling for the direct definition of ordered arrays of apertures with sub-wavelength dimensions and submicron periodicity on the tip of gold-coated fibers [171]. The fiber-optic sensors were formed by coating the prepared tips of the optical fibers with an optically thick layer of gold via electron-beam evaporation and then using FIB milling to fabricate the array of subwavelength apertures. Interaction of light with sub-wavelength structures such as the array of nanoapertures in an optically thick metallic film leads to the excitation of surface plasmon waves at the interfaces of the metallic film and the surrounding media, thereby leading to a significant enhancement of light at certain wavelengths. The realized plasmonic device was proposed for chemical and biological detection. The spectral position and magnitude of the peaks in the transmission spectra depend on the RI of the media surrounding the metallic film containing the nanohole array, enabling the detection of the presence of chemical and biological molecules in the vicinity of the gold film.

An e-beam lithography nano-fabrication process was instead employed by Lin et al. enabling the direct patterning of periodic gold nanodot arrays on optical fiber tips [172]. EBL lithography was preferred with respect to the FIB because FIB milling of gold layers results in unwanted doping of silica and gold with gallium ions [172]. A cleaved fiber was firstly coated by a nanometer thick gold layer by vacuum sputtering methods (a few nm thick $\mathrm{Cr}$ layer was also used as an adhesion layer) followed by the deposition of an electron-beam resist. To this aim, a "dip and vibration" coating technique was exploited enabling to achieve a uniform thickness coating layer (at least in an area large enough to cover the optical fiber mode) on the fiber facet. Then, the EBL process was used to create the two-dimensional nanodot array pattern in the e-beam resist that was successively transferred to the Au layer by RIE etch with Argon ions. The remaining resist was finally striped by dipping the fiber tip in the resist developer. The LSPR of the e-beam patterned gold nanodot arrays on optical fiber tips was then utilized for biochemical sensing [172].

\subsubsection{Hybrid metallo-dielectric nanostructures directly realized on the fiber facet}

Our group also recently introduced a reliable fabrication process that enables the integration of dielectric and metallic nanostructures on the tip of standard optical fibers [16]. It involves conventional deposition and nano-patterning techniques, typically used for planar devices, suitably adapted to directly operate on the optical fiber tip.

As schematic represented in Fig. 5(a), the fabrication process essentially consists of three main technological steps [16]: (1) spin coating deposition of electron-beam resist with accurate thickness control and flat surface over the fiber core region; (2) EBL nano-patterning of the deposited resist; (3) superstrate deposition of different functional materials, either metallic or non-metallic, by using standard coating techniques (such as sputtering, thermal evaporation, etc.). One of the main peculiarities of this approach relies on the capability to deposit dielectric layers (and in particular electron-beam resist like ZEP 520-A) on the cleaved end of optical fibers with controllable thickness (in the range $200 \mathrm{~nm}-400 \mathrm{~nm}$ ) and flat surface over an area of $50 \mathrm{~nm}-60 \mu \mathrm{m}$ in diameter in correspondence of the fiber core. It allows rapid prototyping with a $90 \%$ yield, thanks to the reliable spin coating process which makes the substantial difference with respect to the other fabrication processes reported so far; moreover, our nanostructures show good adhesive strength also resulting in reusable devices.

To attest the capability of the proposed fabrication process, we focused the attention on a first technological platform, realized on the tip of a standard single mode fiber, and based on a 
two-dimensional (2D) hybrid metallo-dielectric nanostructure supporting LSPRs [16].

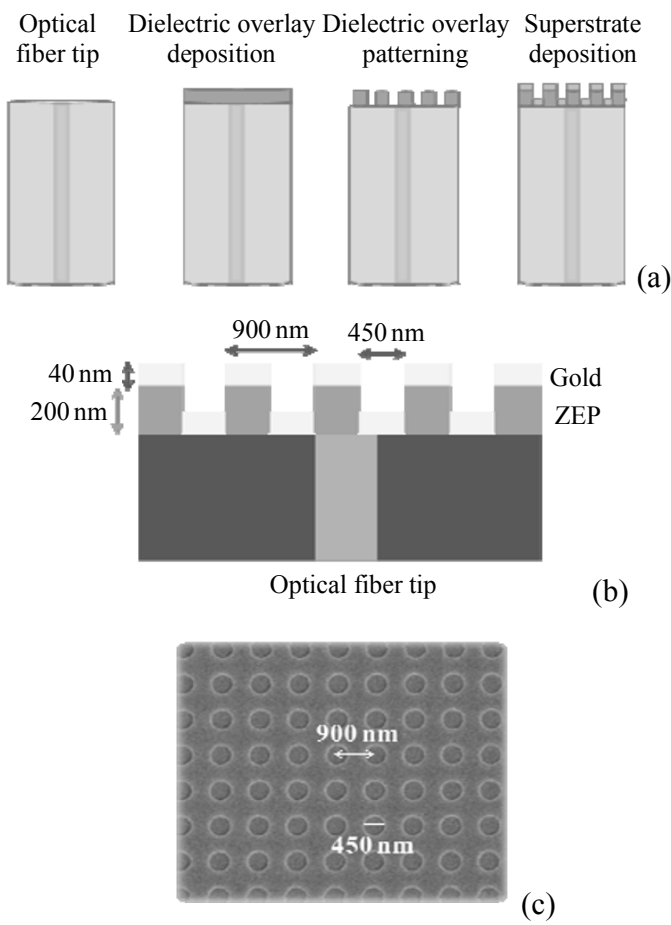

Fig. 5 Hybrid metallo-dielectric nanostructure realized on the tip of a standard single-mode fiber (a) schematic of the main technological steps involved in the fabrication process, (b) cross section schematic view of the hybrid metallo-dielectric nanostructure, and (c) SEM image of the realized hybrid nanostructure [16].

The realized platform demonstrated its feasibility to be used for label free chemical and biological sensing and as a microphone for acoustic wave detection [16]. With reference to Fig. 5(b), it mainly consists of a $200 \mathrm{~nm}$ thick ZEP layer patterned with a square lattice of holes and covered with a $40 \mathrm{~nm}$ thick gold film deposited on both the ridges and the grooves. The lattice period was $a=$ $900 \mathrm{~nm}$, and the holes radius was $r=225 \mathrm{~nm}$. In Fig. 5(c), we show an SEM image of the fabricated hybrid nanostructure.

When the realized nanostructure is illuminated in out-of-plane configuration, as the case of single mode fiber illumination in the paraxial propagation regime, plasmonic and photonic resonances are expected to be excited due to the phase matching condition between the scattered waves and the modes supported by the hybrid structure [173].

From a numerical analysis, carried out by means of the commercial software COMSOL Multiphysics and based on the finite element method, it was shown that the resulting structure supports a transverse electromagnetic wave emulating the normally-incident plane-wave [16]. Figure 6(a) shows the numerically retrieved reflectance spectrum for the structure exhibiting period $a=$ $900 \mathrm{~nm}$ and holes radius $r=225 \mathrm{~nm}$, corresponding to a filling factor of about 0.25 . The high reflectivity $(>85 \%)$ baseline is interrupted by a resonance dip centered at $1369 \mathrm{~nm}$ with a $Q$-factor of about 47 . In the inset of Fig. 6(a), the electric field distribution corresponding to the resonant mode evaluated at the resonance wavelength is shown.

To demonstrate the LSPR resonance tunability, we designed and fabricated two more samples with different periods $(850 \mathrm{~nm}$ and $1000 \mathrm{~nm})$ and the same filling factor $(r=213 \mathrm{~nm}$ and $r=250 \mathrm{~nm})$. Since the resonant wavelengths are directly related to the lattice period, a red (blue) shift is expected in the case of higher (smaller) lattice pitch. The reflectance spectra of the structures with different periods are reported in Fig. 6(b). As predicted by numerical simulations, we experimentally observed a red shift of about $100 \mathrm{~nm}$ and a blue shift of about $70 \mathrm{~nm}$ for $a=1000 \mathrm{~nm}$ and $a=850 \mathrm{~nm}$, respectively.

Reported results open up very intriguing scenarios for the development of a novel generation of miniaturized and cost-effective fiber optic nanosensors useful in many applications including physical, chemical and biological sensing. To show the potentiality of the realized platform, in the following we report some preliminary results demonstrating how our nanosensor is able to sense RI variations in the surrounding environment, suitable for label free chemical and biological 
sensing, as well as to detect acoustic waves [16].

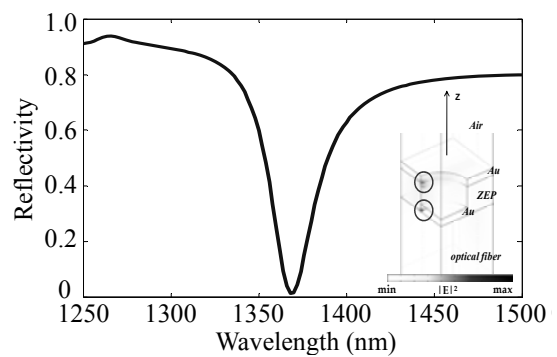

(a)

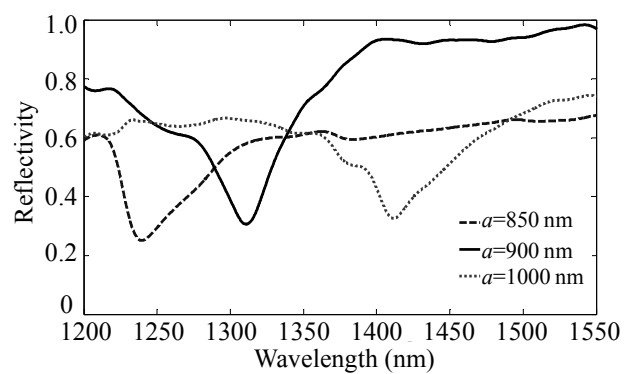

(b)

Fig. 6 Theoretical analysis of hybrid nanostructures realized on the fiber facet: (a) reflectance spectra of the analyzed nanostructure; (inset) 3D view of $1 / 4$ of unit cell and electric field intensity distribution evaluated at the reflectance dip wavelength) and (b) reflectivity of hybrid structures characterized by different periods ( $a=850 \mathrm{~nm}, 900 \mathrm{~nm}$, and $1000 \mathrm{~nm}$ ) [16].

The excitation wavelengths of LSPRs supported by the fabricated nanostructure are very sensitive to variations of the SRI, thus meaning that a change in the local or bulk RI around the fiber tip device gives rise to a wavelength shift in the resonant peak due to a change in the phase matching condition. Actually, a $40 \mathrm{~nm}$ thick gold layer deposited on the top of the fiber tip device strongly shields the external environment from the plasmonic mode excited within the hybrid crystal, resulting in a very weak sensitivity. In order to enhance the surface sensitivity of the final device, it is thus necessary to increase the light matter interaction with the external environment by properly tailoring the resonant mode field distribution. To this aim, all the degrees of freedom exhibited by the hybrid nanostructures can be exploited, e.g. the lattice design and layer thickness [16]. In the following, the results of a sample with a gold layer thickness of only $20 \mathrm{~nm}$ (keeping constant the other geometrical parameters) are shown. The new sample was immersed in different liquid solutions such as water $(n=1.333)$, ethanol $(n=1.362)$ and isopropyl alcohol $(n=1.378)$, and the reflectance spectra were measured by a suitable setup reported in detail in [16]. The experimental results are shown in Fig.7(a), in which the typical red-shift of the curves with increasing values of the SRI can be clearly appreciated. In particular, in the inset of Fig. 7(a), we report the relative wavelength shifts of the dips as a function of the SRI. The graph demonstrates a sensitivity of about $125 \mathrm{~nm} / \mathrm{RIU}$ for detecting changes in the bulk refractive indices of different chemicals surrounding the fiber tip device. We point out that no attempts at this stage have been made to optimize the platform performances. By exploiting the various degrees of freedom offered by the proposed metallo-dielectric nanostructures, some optimization strategies for performance improvement can be pursued.

As a further application, we also investigated the surprising capability of our LSPR-based fiber tip device to detect acoustic waves. Indeed, taking advantage of the typical low Young's modulus of the patterned ZEP, significant variations in the geometrical characteristics of the patterned dielectric slab are expected in response to an applied acoustic pressure wave, hence promoting a consequent shift in the resonant wavelength. As proof of principle, preliminary acoustic experiments have been carried out using a sample characterized by a period $a=$ $900 \mathrm{~nm}$ [whose reflectance spectrum is shown in Fig. 6(b)]. The used experimental setup is reported in [16]. To gather information about the actual incident acoustic pressure, a reference microphone was placed in close proximity of the fiber sensor. In Fig. 7(b) (upper curve), the typical response of the fiber nanodevice to a $4-\mathrm{kHz}$ acoustic tone with a duration of about $250 \mathrm{~ms}$ is reported. For comparison, the response of the reference microphone is also reported in Fig. 7(b) (lower curve). Data clearly reveal the capability of the fiber nanosensor to 
detect acoustic waves in good agreement with the reference microphone. The electrical signal is delayed in respect to the optical counterpart, due to the slightly longer distance at which the reference microphone is located from the acoustic source as well as to the different electronic processing applied to the acoustic signal with respect to the optical one. It is worth noting that, although a relatively high noise level is visible in Fig. 7(b) (the standard deviation of the sensor signal $-\sigma_{\text {noise }}-$ in absence of the acoustic wave is nearly $0.1 \mathrm{~V}$ ), attributable to the instability of the utilized tunable laser, the response of the metallo-dielectric fiber device was found to be more than an order of magnitude higher than the noise level.

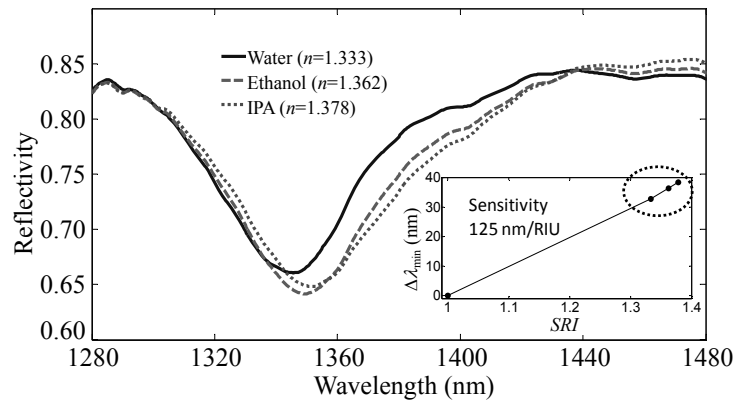

(a)

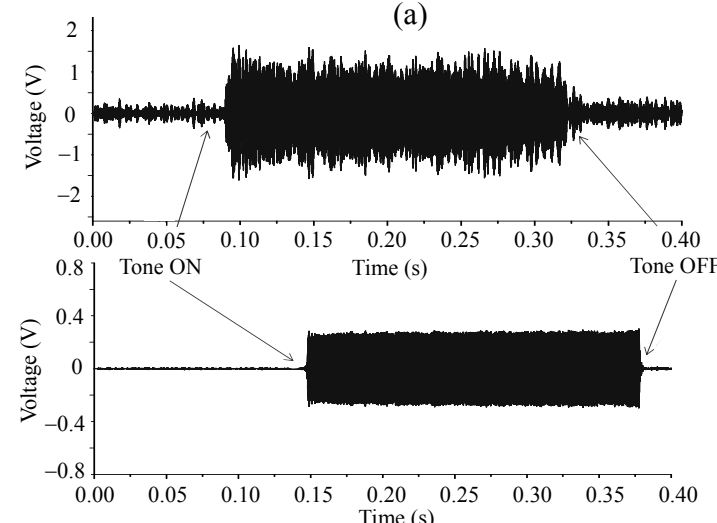

(b)

Fig. 7 Preliminary experimental results demonstrating the capability of the hybrid fiber tip device to be used for different sensing applications: (a) reflectance spectra of a sample with a $20 \mathrm{~nm}$ thick gold layer, immersed in water (solid), ethanol (dashed) and isopropyl alcohol (dotted); (inset) relative wavelength shifts in the reflection dips as a function of the SRI and (b) typical time responses of the hybrid metallo-dielectric fiber tip device (top) and reference microphone (bottom) to a $4-\mathrm{kHz}$ acoustic pressure pulse with a duration of $250 \mathrm{~ms}$ [16].
The above results are only preliminary, and no efforts have been made to optimize the performance of the final device. Also in this case, further optimization margins exist by properly tailoring the nanostructure design to maximize the dependence of the resonant wavelength on the geometric features of the patterned polymer.

\section{Conclusions}

Ever since optical fibers have been conceived as reliable and high performances communication media, in less than half a century, optical fiber technology has driven the communication revolution and nowadays represents one of the key elements of the global communication network. In the last decade, two significant advancements have characterized optical fiber technology:

(1) The development of novel optical fiber platforms (MOF, POF, HOF,..).

(2) The development of multifunctional and multimaterial devices integrated in the optical fiber.

All these collective efforts can be viewed as concurring to the lab-on-fiber realization. The basic idea of the lab-on-fiber relies on the development of a new technological world completely included in a single optical fiber where several structures and materials at nano- and micro-scale are constructed, embedded and connected all together to provide the necessary physical connections and light-matter interactions useful to provide a wide range of functionalities for specific communication and sensing applications.

The research efforts carried out in this direction in the last decade have confirmed the potentiality of the lab-on-fiber concept through feasibility analysis devoted to identify and define technological scenarios for the fabrication of highly functionalized all-in-fiber components, devices and systems for different strategic sectors.

In the future scenario, it can be envisioned that a novel generation of fiber optic micro and nanodevices for sensing and communication 
applications could arise through the concurrent addressing of the issues related to the different aspects of the lab-on-fiber global design which requires highly integrated and multidisciplinary competencies, ranging from photonics to physics, from material science to biochemistry, up to micro and nanotechnologies. Moreover, a highly integrated approach involving continuous interactions of different backgrounds is required to optimize each single aspect with a continuous feed-back that would enable the definition of the overall and global design.

Open Access This article is distributed under the terms of the Creative Commons Attribution License which permits any use, distribution, and reproduction in any medium, provided the original author(s) and source are credited.

\section{References}

[1] P. Russell, "Photonic crystal fibers," Science, vol. 299, no. 5605, pp. 358-362, 2003.

[2] J. C. Knight, "Photonic crystal fibres," Nature, vol. 424, no. 6950, pp. 847-851, 2003.

[3] O. Ziemann, J. Krauser, P. E. Zamzow, and W. Daum, POF handbook: optical short range transmission systems. Berlin, Germany: Springer-Verlag, 2008.

[4] M. A. van Eijkelenborg, M. C. J. Large, A. Argyros, J. Zagari, S. Manos, N. A. Issa, et al., "Microstructured polymer optical fibre," Optics Express, vol. 9, no. 7, pp. 319-327, 2001.

[5] A. Cusano, M. Consales, M. Pisco, A. Crescitelli, A. Ricciardi, E. Esposito, et al., "Lab on fiber technology and related devices, part I: a new technological scenario; lab on fiber technology and related devices, part II: the impact of the nanotechnologies," in Proc. SPIE, vol. 8001, pp. $800122,2011$.

[6] A. Cusano, D. Paladino, and A. Iadicicco, "Microstructured fiber Bragg gratings," Journal of Lightwave Technology, vol. 27, no. 11, pp. 1663-1697, 2009.

[7] A. Cusano, M. Giordano, A. Cutolo, M. Pisco, and M. Consales, "Integrated development of chemoptical fiber nanosensors," Current Analytical Chemistry, vol. 4, no. 4, pp. 296-315, 2008.

[8] J. Canning, "Fibre gratings and devices for sensors and lasers," Laser and Photonics Reviews, vol. 2, no. 4, pp. 275-289, 2008.

[9] B. J. Eggleton, C. Kerbage, P. S. Westbrook, R. S. Windeler, and A. Hale, "Microstructured optical fiber devices," Optics Express, vol. 9, no. 13, pp. 698-713, 2001.

[10] F. J. Arregui, Sensors based on nanostructured materials. New York: Springer, 2009.

[11] B. Lee, S. Roh, and J. Park, "Current status of micro- and nano-structured optical fiber sensors," Optical Fiber Technology, vol. 15, no. 3, pp. 209-221, 2009.

[12] A. F. Abouraddy, M. Bayindir, G. Benoit, S. D. Hart, K. Kuriki, N. Orf, et al., "Towards multimaterial multifunctional fibres that see, hear, sense and communicate," Nature Materials, vol. 6, no. 5, pp. 336-347, 2007.

[13] E. J. Smythe, M. D. Dickey, G. M. Whitesides, and F. A. Capasso, "A technique to transfer metallic nanoscale patterns to small and nonplanar surfaces," ACS Nano, vol. 3, no. 1, pp. 59-65, 2009.

[14] D. J. Lipomi, R. V. Martinez, M. A. Kats, S. H. Kang, P. Kim, J. Aizenberg, et al., "Patterning the tips of optical fibers with metallic nanostructures using nanoskiving," Nano Letters, vol. 11, no. 2, pp. 632-636, 2011.

[15] D. Iannuzzi, S. Deladi, V. J. Gadgil, R. G. P. Sanders, H. Schreuders, and M. C. Elwenspoek, "Monolithic fiber-top sensor for critical environments and standard applications," Applied Physics Letters, vol. 88, no. 5, pp. 053501, 2006.

[16] M. Consales, A. Ricciardi, A. Crescitelli, E. Esposito, A. Cutolo, and A. Cusano, "Lab-on-fiber technology: towards multi-funcional optical nanoprobes," $A C S$ Nano, vol. 6, no. 4, pp. 3163-3170, 2012.

[17] G.. Brambilla, "Optical fibre nanowires and microwires: a review," Journal of Optics, vol. 12, no. 4, pp. 043001, 2010.

[18] J. Canning and M. G. Sceats, " $\pi$-phase-shifted periodic distributed structures in germanosilicate fibre by UV post-processing," Electronics Letters, vol. 30, no. 16, pp. 1344-1345, 1994.

[19] M. Janos and J. Canning, "Permanent and transient resonances thermally induced in optical fibre Bragg gratings," Electronics Letters, vol. 31, no. 12, pp. 1007-1009, 1995.

[20] D. Uttamchandani and A. Othonos, "Phase shifted Bragg gratings formed in optical fibres by post-fabrication thermal processing," Optics Communications, vol. 127, no. 4-6, pp. 200-204, 1996.

[21] A. Iadicicco, A. Cusano, S. Campopiano, A. Cutolo, and M. Giordano, "Microstructured fiber Bragg gratings: analysis and fabrication," Electronics Letters, vol. 41, no. 8, pp. 466-468, 2005.

[22] R. Zengerle and O. Leminger, "Phase-shifted Bragg-grating filters with improved transmission 
characteristics," Journal of Lightwave Technology, vol. 13, no. 12, pp. 2354-2358, 1995.

[23] L. Wei and J. W. Y. Lit, "Phase-shifted Bragg grating filters with symmetrical structures," Journal of Lightwave Technology, vol. 15, no. 8, pp. 1405-1410, 1997.

[24] A. Cusano, A. Iadicicco, S. Campopiano, M. Giordano, and A. Cutolo, "Thinned and micro-structured fiber Bragg gratings: towards new all fiber high sensitivity chemical sensors," Journal of Optics A: Pure and Applied Optics, vol. 7, no. 12, pp. 734-741, 2005.

[25] A. Asseh, S. Sandgren, H. Ahlfeldt, B. Sahlgren, R. Stubbe, and G. Edwall, "Fiber optical Bragg grating refractometer," Fiber and Integrated Optics, vol. 7, no. 1, pp. 51-62, 1998.

[26] A. Iadicicco, S. Campopiano, D. Paladino, A. Cutolo, and A. Cusano, "Micro-structured fiber Bragg gratings: optimization of the fabrication process," Optics Express, vol. 15, no. 23, pp. 15011-15021, 2007.

[27] A. Cusano, A. Iadicicco, D. Paladino, S. Campopiano, and A. Cutolo, "Photonic band-gap engineering in UV fiber gratings by the arc discharge technique," Optics Express, vol. 16, no. 20, pp. 15332-15342, 2008.

[28] D. Paladino, A. Iadicicco, S. Campopiano, and A. Cusano, "Not-lithographic fabrication of micro-structured fiber Bragg gratings evanescent wave sensors," Optics Express, vol. 17, no. 2, pp. 1042-1054, 2009.

[29] W. C. Du, X. M. Tao, and H. Y. Tam, "Fiber Bragg grating cavity sensor for simultaneous measurement of strain and temperature," IEEE Photonics Technology Letters, vol. 11, no. 1, pp. 105-107, 1999.

[30] K. Zhou, Y. Lai, X. Chen, K. Sugden, L. Zhang, and I. Bennion, "A refractometer based on a micro-slot in a fiber Bragg grating formed by chemically assisted femtosecond laser processing," Optics Express, vol. 15, no. 24, pp. 15848-15853, 2007.

[31] M. Pisco, A. Iadicicco, S. Campopiano, A. Cutolo, and A. Cusano, "Structured chirped fiber Bragg gratings," Journal of Lightwave Technology, vol. 26, no. 12, pp. 1613-1625, 2008.

[32] S. W. James and R. P. Tatam, "Optical fibre long period grating sensors: characteristics and application," Measurement Science and Technology, vol. 14, no. 5, pp. R49-R61, 2003.

[33] N. D. Rees, S. W. James, R. P. Tatam, and G. J. Ashwell, "Optical fibre long period gratings with Langmuir-Blodgett thin-film overlays," Optics Letters, vol. 27, no. 9, pp. 686-688, 2002.

[34] I. Del Villar, M. Achaerandio, I. R. Matias, and F. J. Arregui, "Deposition of overlays by electrostatic self assembly in long-period fiber gratings," Optics Letters, vol. 30, no. 7, pp. 720-722, 2005.

[35] I. Del Villar, I. R. Matias, F. J. Arregui, and
P. Lalanne, "Optimization of sensitivity in long period fiber gratings with overlay deposition," Optics Express, vol. 13, no. 1, pp. 56-69, 2005.

[36] P. Pilla, A. Iadicicco, L. Contessa, S. Campopiano, A. Cutolo, M. Giordano, et al., "Optical chemosensor based on long period gratings coated with $\delta$ form syndiotactic polystyrene," IEEE Photonics Technology Letters, vol. 17, no. 8, pp. 1713-1715, 2005.

[37] A. Cusano, A. Iadicicco, P. Pilla, L. Contessa, S. Campopiano, A. Cutolo, et al., "Cladding modes re-organization in high refractive index coated long period gratings: effects on the refractive index sensitivity," Optics Letters, vol. 30, no. 9, pp. 2536-25387, 2005.

[38] E. Simões, I. Abe, J. Oliveira, O. Frazão, P. Caldas, and J. L. Pinto, "Characterization of optical fiber long period grating refractometer with nanocoating," Sensors and Actuators B: Chemical, vol. 153, no. 2, pp. 335-339, 2011.

[39] A. Cusano, A. Iadicicco, P. Pilla, L. Contessa, S. Campopiano, A. Cutolo, et al., "Mode transition in high refractive index coated long period gratings," Optics Express, vol. 14, no. 1, pp, 19-34, 2006.

[40] P. Pilla, A. Cusano, A. Cutolo, M. Giordano, G. Mensitieri, P. Rizzo, et al., "Molecular sensing by nanoporous crystalline polymers," Sensors, vol. 9, no. 12, pp. 9816-9857, 2009.

[41] N. D. Rees, S. W. James, R. P. Tatam, and G. J. Ashwell, "Optical fiber long-period gratings with Langmuir-Blodgett thin-film overlays," Optics Letters, vol. 27, no. 9, pp. 686-688, 2002.

[42] A. Cusano, P. Pilla, L. Contessa, A. Iadicicco, S. Campopiano, A. Cutolo, et al., "High sensitivity optical chemosensor based on coated long-period gratings for sub-ppm chemical detection in water," Applied Physics Letters, vol. 87, no. 23, pp. 234105-1-234105-3, 2005.

[43] Z. Gu and Y. Xu, "Design optimization of a long-period fiber grating with sol-gel coating for a gas sensor," Measurement Science and Technology, vol. 18, no. 11, pp. 3530-3536, 2007.

[44] D. Viegas, J. Goicoechea, J. L. Santos, F. M. Araújo, L. A. Ferreira, F. J. Arregui, et al., "Sensitivity improvement of a humidity sensor based on silica nanospheres on a long-period fiber grating," Sensors, vol. 9, no. 1, pp. 519-527, 2009.

[45] J. M. Corres, I. R. Matias, I Del Villar, and F. J. Arregui, "Design of $\mathrm{pH}$ sensors in long-period fiber gratings using polymeric nanocoatings," IEEE Sensors Journal, vol. 7, no. 3, pp. 455-463, 2007.

[46] M. Konstantaki, S. Pissadakis, S. Pispas, N. Madamopoulos, and N. A. Vainos, "Optical fiber long-period grating humidity sensor with poly(ethylene oxide)/cobalt chloride coating," Applied Optics, vol. 45, no. 19, pp. 4567-4571, 2006. 
[47] D. Viegas, J. Goicoechea, J. M. Corres, J. L. Santos, L. A. Ferreira, F. M. Araújo, et al., "A fiber optic humidity sensor based on a long-period fiber grating coated with a thin film of $\mathrm{SiO}_{2}$ nanospheres," Measurement Science and Technology, vol. 20, no. 3, pp. 034002, 2009.

[48] A. Cusano, A. Iadicicco, P. Pilla, A. Cutolo, M. Giordano, and S. Campopiano, "Sensitivity characteristics in nanosized coated long period gratings," Applied Physics Letters, vol. 89, no. 20, pp. 201116-1-201116-3, 2006.

[49] S. James and R. Tatam, "Fiber optic sensors with nano-structured coatings," Journal of Optics A: Pure and Applied Optics, vol. 8, no. 7, pp. S430-S444, 2006.

[50] P. Pilla, P. Foglia Manzillo, V. Malachovska, A. Buosciolo, S. Campopiano, A. Cutolo, et al., "Long period grating working in transition mode as promising technological platform for label-free biosensing," Optics Express, vol. 17, no. 22, pp. 20039-20050, 2009.

[51] P. Pilla, V. Malachovska, A. Borriello, A. Buosciolo, M. Giordano, L. Ambrosio, et al., "Transition mode long period grating biosensor with functional multilayer coatings," Optics Express, vol. 19, no. 2, pp, 512-526, 2011

[52] P. Foglia Manzillo, P. Pilla, A. Buosciolo, S. Campopiano, A. Cutolo, A. Borriello, et al., "Self assembling and coordination of water nano-layers on polymer coated long period gratings: toward new perspectives for cation detection," Soft Materials, vol. 9, no. 2-3, pp. 238-263, 2011.

[53] G. Meltz, S. J. Hewlett, and J. D. Love, "Fiber grating evanescent wave sensors," in Proc. SPIE, vol. 2836, pp. 342-350, 1996.

[54] D. J. Markos, B. L. Ipson, K. H. Smith, S. M. Schultz, and R. H. Selfridge, "Controlled core removal from a D-shaped optical fiber," Applied Optics, vol. 42, no. 36, pp. 7121-7125, 2003.

[55] T. L. Lowder, K. H. Smith, B. L. Ipson, A. R. Hawkins, R. H. Selfridge, and S. M. Schultz, "High-temperature sensing using surface relief fiber bragg gratings," IEEE Photonics Technology Letters, vol. 17, no. 9, pp. 1926-1928, 2005.

[56] R. H. Selfridge, S. M. Schultz, T. L. Lowder, V. P. Wnuk, A. Mendez, S. Ferguson, et al., "Packaging of surface relief fiber bragg gratings for use as strain sensors at high temperature," in Proc. SPIE, vol. 6167, pp. 616702-1-616702-7, 2006.

[57] T. L. Lowder, J. D. Gordon, S. M. Schultz, and R. H. Selfridge, "Volatile organic compound sensing using a surface relief d-shaped fiber Bragg grating and a polydimethylsiloxane layer," Optics Letters, vol. 32, no. 17, pp. 2523-2525, 2007.

[58] H. S. Jang, K. N. Park, J. P. Kim, O. J. Kwon, Y. G. Han, and K. S. Lee, "Sensitive DNA biosensor based on a long-period grating formed on the side-polished fiber surface," Optics Express, vol. 17, no. 5, pp. 3855-3860, 2009.

[59] G. Quero, A. Crescitelli, D. Paladino, M. Consales, A. Buosciolo, M. Giordano, et al., "Evanescent wave long-period fiber grating within D-shaped optical fibers for high sensitivity refractive index detection," Sensors and Actuators B: Chemical, vol. 152, no. 2, pp. 196-205, 2011.

[60] X. Shu, L. Zhang, and I. Bennion, "Sensitivity characteristics of long-period fiber gratings," Journal of Lightwave Technology, vol. 20, no. 2, pp. 255-266, 2002.

[61] L. Rindorf and O. Bang, "Highly sensitive refractometer with a photonic-crystal-fiber long-period grating," Optics Letters, vol. 33, no. 5, pp. 563-565, 2008.

[62] J. Kong, N. R. Franklin, C. Zhou, M. G. Chapline, S. Peng, K. Cho, et al., "Nanotube molecular wires as chemical sensors," Science, vol. 287, no. 5453, pp. 622-625, 2000.

[63] M. Penza, G. Cassano, P. Aversa, F. Antolini, A. Cusano, A. Cutolo, et al., "Alcohol detection using carbon nanotubes acoustic and optical sensors," Applied Physics Letters, vol. 85, no. 12, pp. 2378-2381, 2004.

[64] M. Penza, G. Cassano, P. Aversa, A. Cusano, A. Cutolo, M. Giordano, et al., "Carbon nanotube acoustic and optical sensors for volatile organic compound detection," Nanotechnology, vol. 16, no. 11, pp. 2536-2547, 2005.

[65] M. Consales, A. Cutolo, M. Penza, P. Aversa, G. Cassano, M. Giordano, et al., "Carbon nanotubes coated acoustic and optical VOCs sensors: towards the tailoring of the sensing performances," IEEE Transactions on Nanotechnology, vol. 6, no. 6, pp. 601-612, 2007.

[66] M. Consales, S. Campopiano, A. Cutolo, M. Penza, P. Aversa, G. Cassano, et al., "Carbon nanotubes thin films fiber optic and acoustic VOCs sensors: performances analysis," Sensors and Actuators B: Chemical, vol. 118, no. 1-2, pp. 232-242, 2006.

[67] M. Consales, A. Crescitelli, M. Penza, P. Aversa, P. Delli Veneri, M. Giordano, et al., "SWCNT nano-composite optical sensors for VOC and gas trace detection," Sensors and Actuators B: Chemical, vol. 138, no. 1, pp. 351-361, 2009.

[68] M. Consales, A. Crescitelli, S. Campopiano, A. Cutolo, M. Penza, P. Aversa, et al., "Chemical detection in water by single-walled carbon nanotubes-based optical fiber sensors," IEEE Sensors Journal, vol. 7, no. 7, pp. 1004-1005, 2007.

[69] A. Cusano, M. Consales, A. Cutolo, M. Penza, P. Aversa, M. Giordano, et al., "Optical probes based on optical fibers and single-walled carbon nanotubes for hydrogen detection at cryogenic temperatures," Applied Physics Letters, vol. 89, no. 20, pp. 201106-1-201106-3, 2007. 
[70] S. Y. Set, H. Yaguchi, Y. Tanaka, and M. Jablonski, "Ultrafast fiber pulsed lasers incorporating carbon nanotubes," IEEE Journal of Selected Topics in Quantum Electronics, vol. 10, no. 1, pp. 137-146, 2004.

[71] S. Yamashita, Y. Inoue, S. Maruyama, Y. Murakami, H. Yaguchi, M. Jablonski, et al., "Saturable absorbers incorporating carbon nanotubes directly synthesized onto substrates/fibers and their applications to mode-locked fiber lasers," Optics Letters, vol. 29, no. 14, pp. 1581-1583, 2004.

[72] K. Kashiwagi and S. Yamashita, "Optically manipulated deposition of carbon nanotubes onto optical fiber end," Japanese Journal of Applied Physics, vol. 46, no. 40, pp. L988-L990, 2007.

[73] K. K. Chow and S. Yamashita, "Four-wave mixing in a single-walled carbon-nanotube-deposited D-shaped fiber and its application in tunable wavelength conversion," Optics Express, vol. 17, no. 18 , pp. 15608-15613, 2009.

[74] G. Sberveglieri, "Recent developments in semiconducting thin-film gas sensors," Sensors and Actuators B: Chemical, vol. 23, no. 2-3, no. 103-109, 1995.

[75] M. Batzill and U. Diebold, "The surface and materials science of tin oxide," Progress in Surface Science, vol. 79, no. 24, pp. 47-154, 2005.

[76] M. Pisco, M. Consales, S. Campopiano, A. Cutolo, R. Viter, V. Smyntyna, et al., "A novel opto-chemical sensor based on $\mathrm{SnO}_{2}$ sensitive thin film for ppm ammonia detection in liquid environment," Journal of Lightwave Technology, vol. 24, no. 12, pp. 5000-5007, 2006.

[77] A. Cusano, M. Consales, M. Pisco, P. Pilla, A. Cutolo, A. Buosciolo, et al., "Opto-chemical sensor for water monitoring based on $\mathrm{SnO}_{2}$ particle layer deposited onto optical fibers by the electrospray pyrolysis method," Applied Physics Letters, vol. 89, no. 11, pp. 111103-1-111103-3, 2006.

[78] A. Buosciolo, M. Consales, M. Pisco, A. Cusano, and M. Giordano, "Fiber optic near-field chemical sensors based on wavelength scale tin dioxide particle layers," Journal of Lightwave Technology, vol. 26, no. 20, pp. 3468-3475, 2008.

[79] A. Cusano, P. Pilla, M. Consales, M. Pisco, A. Cutolo, A. Buosciolo, et al., "Near field behavior of $\mathrm{SnO}_{2}$ particle-layer deposited on standard optical fiber by electrostatic spray pyrolysis method," Optics Express, no. 15 , no. 8, pp. 5136-5146, 2007.

[80] M. Fossa and P. Petagna, "Use and calibration of capacitive RH sensors for the hygrometric control of the CMS tracker," CMS NOTE2003/24, Cern, Geneve, Switzerland, 2003.

[81] M. Consales, A. Buosciolo, A. Cutolo, G. Breglio, A. Irace, S. Buontempo, et al., "Fiber optic humidity sensors for high-energy physics application at
CERN," Sensors and Actuators B: Chemical, vol. 159, no. 1, pp 66-74, 2011.

[82] M. C. Phan Huy, G. Laffont, Y. Frignac, V. Dewynter-Marty, P. Ferdinand, P. Roy, et al., "Fibre Bragg grating photowriting in microstructured optical fibres for refractive index measurement," Measurement Science and Technology, vol. 17, no. 5, pp. 992-997, 2006.

[83] M. C. Phan Huy, G. Laffont, V. Dewynter, P. Ferdinand, P. Roy, J. L. Auguste, et al., "Three-hole microstructured optical fiber for efficient fiber Bragg grating refractometer," Optics Letters, vol. 32, no. 16, pp. 2390-2392, 2007.

[84] L. Rindorf, P. E. Hoiby, J. B. Jensen, L. H. Pedersen, O. Bang, and O. Geschke, "Towards biochips using microstructured optical fiber sensors," Analytical and Bioanalytical Chemistry, vol. 385, no. 8, pp. 1370-1375, 2006.

[85] C. M. B. Cordeiro, M. A. R. Franco, G. Chesini, E. C. S. Barretto, R. Lwin, C. H. B. Cruz, et al., "Microstructured-core optical fibre for evanescent sensing applications," Optics Express, vol. 14, no. 26, pp. 13056-13066, 2006.

[86] Y. Huang, Y. Xu, and A. Yariv, "Fabrication of functional microstructured optical fibers through a selective-filling technique," Applied Physics Letters, vol. 85, no. 22, pp. 5182-5184, 2004.

[87] S. Smolka, M. Barth, and O. Benson, "Highly efficient fluorescence sensing with hollow core photonic crystal fibers," Optics Express, vol. 15, no. 20, pp. 12783-12791, 2007.

[88] J. B. Jensen, P. E. Hoiby, G. Emiliyanov, O. Bang, L. H. Pedersen, and A. Bjarklev, "Selective detection of antibodies in microstructured polymer optical fibers," Optics Express, vol. 13, no. 15, pp. 5883-5889, 2005.

[89] S. Smolka, M. Barth, and O. Benson, "Selectively coated photonic crystal fiber for highly sensitive fluorescence detection," Applied Physics Letters, vol. 90, no. 11, pp. 111101, 2007.

[90] S. O. Konorov, A. M. Zheltikov, and M. Scalora, "Photonic-crystal fiber as a multifunctional optical sensor and sample collector," Optics Express, vol. 13, no. 9 , pp. 3454-3459, 2005.

[91] S. Afshar v., S. C. Warren-Smith, and T. M. Monro, "Enhancement of fluorescence-based sensing using microstructured optical fibres," Optics Express, vol. 15, no. 26, pp. 17891-17901, 2007.

[92] T. Ritari, J. Tuominen, H. Ludvigsen, J. Petersen, T. Sørensen, T. Hansen, et al., "Gas sensing using air-guiding photonic bandgap fibers," Optics Express, vol. 12, no. 17, pp. 4080-4087, 2004.

[93] Y. Ruan, T. C. Foo, St. Warren-Smith, P. Hoffmann, R. C. Moore, H. Ebendorff-Heidepriem, et al., "Antibody immobilization within glass microstructured fibers: a route to sensitive and selective biosensors," Optics Express, vol. 16, no. 22, 
pp. 18514-18523, 2008.

[94] J. Canning, "Structured optical fibres and the application of their linear and non-linear properties," in Selected topics in photonic crystals and metamaterials, A. Andreone, A. Cusano, A. Cutolo, and V. Galdi, Eds. Singapore: World Scientific Publishing Co. Pte. Ltd., 2011, pp. 389-452.

[95] T. Larson, J. Broeng, D. Hermann, and A. Bjarklev, "Thermo-optic switching in liquid crystal infiltrated photonic bandgap fibres," Electronics Letters, vol. 39, no. 24, pp. 1719-1720, 2003.

[96] T. Larson, A. Bjarklev, D. Hermann, and J. Broeng, "Optical devices based on liquid crystal photonic bandgap fibres," Optics Express, vol. 11, no. 20, pp. 2589-2596, 2003.

[97] M. Haakestad, M. Alkeskjold, M. Nielsen, L. Scolari, J. Riishede, H. Engan, et al., "Electrically tuneable photonic bandgap guidance in a liquid-crystal-filled photonic crystal fibre," IEEE Photonics Technology Letters, vol. 17, no. 4, pp. 819-821, 2005.

[98] J. Hou, D. Bird, A. George, S. Maier, B. T. Kuhlmey, and J. C. Knight, "Metallic mode confinement in microstructured fibres," Optics Express, vol. 16, no. 9, pp. 5983-5990, 2008.

[99] C. Grillet, P. Domachuk, V. Taeed, E. Mägi, J. Bolger, B. Eggleton, et al., "Compact tunable microfluidic interferometer," Optics Express, vol. 12, no. 24, pp. 5440-5447, 2004.

[100] A. Cusano, M. Pisco, M. Consales, A. Cutolo, M. Giordano, M. Penza, et al., "Novel opto-chemical sensors based on hollow fibers and single walled carbon nanotubes," IEEE Photonics Technology Letters, vol. 18, no. 22, pp. 2431-2433, 2006.

[101] M. Pisco, M. Consales, A. Cutolo, M. Penza, P. Aversa, and A. Cusano, "Hollow fibers integrated with single walled carbon nanotubes: bandgap modification and chemical sensing capability," Sensors and Actuators B: Chemical, vol. 129, no. 1, pp. 163-170, 2008.

[102] C. Kerbage, R. S. Windeler, B. J. Eggleton, P. Mach, M. Dolinski, and J. A. Rogers, "Tunable devices based on dynamic positioning of micro-fluids in micro-structured optical fiber," Optics Communications, vol. 204, no. 1-6, pp. 179-184, 2002.

[103] C. Martelli, P. Olivero, J. Canning, N. Groothoff, B. Gibson, and S. Huntington, "Micromachining structured optical fibers using focused ion beam milling," Optics Letters, vol. 32, no. 11, pp. 1575-1577, 2007.

[104] S. Yiou, P. Delaye, A. Rouvie, J. Chinaud, R. Frey, G. Roosen, et al., "Stimulated Raman scattering in an ethanol core microstructured optical fiber," Optics Express, vol. 13, no. 12, pp. 4786-4791, 2005.

[105] K. Nielsen, D. Noordegraaf, T. Sørensen, A. Bjarklev, and T. P., Hansen, "Selective filling of photonic crystal fibres," Journal of Optics A: Pure and Applied Optics, vol. 7, no. 8, pp. L13-L20, 2005.

[106] L. Xiao, W. Jin, M. S. Demokan, H. L. Ho, Y. L. Hoo, and C. Zhao, "Fabrication of selective injection microstructured optical fibers with a conventional fusion splicer," Optics Express, vol. 13, no. 22, pp. 9014-9022, 2005.

[107] C. J. De Matos, C. M. B. Cordeiro, E. M. Dos Santos, J. S. Ong, A. Bozolan, and C. H. B. Cruz, "Liquid-core, liquid-cladding photonic crystal fibers," Optics Express, vol. 15, no. 18, pp. 11207-11212, 2007.

[108] J. Canning, M. Stevenson, T. K. Yip, S. K. Lim, and C. Martelli, "White light sources based on multiple precision selective micro-filling of structured optical waveguides," Optics Express, vol. 16, no. 20, pp. 15700-15708, 2008.

[109] Y. Han and H. Du, "Photonic crystal fiber for chemical sensing using surface-enhanced Raman scattering," in Photonic Bandgap Structures: Novel Technological Platforms for Physical, Chemical and Biological Sensing. M. Pisco, A. Cusano and, A. Cutolo, Ed. Oak Park, IL: Bentham Science Publisher, 2012, pp. 157-179.

[110] X. Yang, C. Shi, R. Newhouse, J. Z. Zhang, and C. $\mathrm{Gu}$, "Hollow-core photonic crystal fibers for surfaceenhanced Raman scattering probes," International Journal of Optics, vol. 2011 (article ID 754610), pp 754610-1-754610-11, 2011.

[111] H. Yan, J. Liu, C. Yang, G. Jin, C. Gu, and L. Hou, "Novel index-guided photonic crystal fiber surface-enhanced Raman scattering probe," Optics Express, vol. 16, no. 11, pp. 8300-8305, 2008.

[112] A. Amezcua-Correa, J. Yang, and C. E. Finlayson, "Surface-enhanced Raman scattering using microstructured optical fiber substrates," Advanced Functional Materials, vol. 17, no. 13, pp. 2024-2030, 2007.

[113] M. K. Khaing Oo, Y. Han, R. Martini, S. Sukhishvili, and H. Du, "Forward-propagating surface-enhanced Raman scattering and intensity distribution in photonic crystal fiber with immobilized $\mathrm{Ag}$ nanoparticles," Optics Letters, vol. 34, no. 7, pp. 968-970, 2009.

[114] M. K. Khaing Oo, Y. Han, J. Kanka, S. Sukhishvili, and H. Du, "Structure fits the purpose: photonic crystal fibers for evanescent-field surface-enhanced Raman spectroscopy," Optics Letters, vol. 35, no. 4, pp. 466-468, 2010.

[115] Y. Han, S. Tan, M. K. Khaing Oo, D. Pristinski, S. Sukhishvili, and H. Du, "Towards full-length accumulative surface-enhanced Raman scattering-active photonic crystal fibers," Advanced Materials, vol. 22, no. 24, pp. 2647-2651, 2010.

[116] G. Whitesides, J. Kriebel, and B. Mayers, "Self-assembly and nanostructured materials," in 
Nanoscale Assembly: Chemical techniques. B. T. Mayers, Ed. New York: Springer US, 2009, pp. 217-239.

[117] F. J. Arregui, I. R. Matias, J. M. Corres, I. Del Villar, J. Goicoechea, C. R. Zamarreño, et al. "Optical fiber sensors based on layer-by-layer nanostructured films," Procedia Engineering, vol. 5, pp. 1087-1090, 2010.

[118] I. Del Villar, I. R. Matias, and F. J. Arregui, "Fiber-optic chemical nanosensors by electrostatic molecular self-assembly," Current Analytical Chemistry, vol. 4, no. 4, pp. 341-355, 2008.

[119] J. Homola, S. S. Yeea, and G. Gauglitz, "Surface plasmon resonance sensors: review," Sensors and Actuators B: Chemical, vol. 54, no. 1-2, pp. 3-15, 1999.

[120] J. Homola, "Surface plasmon resonance sensors for detection of chemical and biological species," Chemical Reviews, vol. 108, no. 2, pp. 462-493, 2008.

[121] M. E. Stewart, C. R. Anderton, L. B. Thompson, J. Maria, S. K. Gray, J. A. Rogers, et al., "Nanostructured plasmonic sensors," Chemical Reviews, vol. 108, no. 2, pp. 494-521, 2008.

[122] S. Roh, T. Chung, and B. Lee, "Overview of the characteristics of micro- and nano-structured surface plasmon resonance sensors," Sensors, vol. 11, no. 2, pp. 1565-1588, 2011.

[123] A. K. Sharma and B. D. Gupta, "Fibre-optic sensor based on surface plasmon resonance with Ag-Au alloy nanoparticle films," Nanotechnology, vol. 17, no. 1, pp. 124-131, 2006.

[124] M. Kanso, S. Cuenot, and G. Louarn, "Sensitivity of optical fiber sensor based on surface plasmon resonance: Modeling and experiments," Plasmonics, vol. 3, no. 2-3, pp. 49-57, 2008.

[125] E. M. Yeatman, "Resolution and sensitivity in surface plasmon microscopy and sensing," Biosensors and Bioelectronics, vol. 11, no. 6, pp. 635-649, 1996.

[126] J. Homola, I. Koudela, and S. Yee, "Surface plasmon resonance sensor based on diffraction gratings and prism couplers: sensitivity comparison," Sensors and Actuators B: Chemical, vol. 54, no. 1-2, pp. 16-24, 1999.

[127] N. Díaz-Herrera, A. González-Cano, D. Viegas, J. L. Santos, and M. C. Navarrete, "Refractive index sensing of aqueous media based on plasmonic resonance in tapered optical fibres operating in the $1.5 \mu \mathrm{m}$ region," Sensors and Actuators B: Chemical, vol. 146, no. 1, pp. 195-198, 2010.

[128] S. F. Wang, M. H. Chiu, J. C. Hsu, R. S. Chang, and F. T. Wang, "Theoretical analysis and experimental evaluation of D-type optical fiber sensor with a thin gold film," Optics Communications, vol. 253, no. 4-6, pp. 283-289,
2005.

[129] M. H. Chiu and C. H. Shih, "Searching for optimal sensitivity of single-mode D-type optical fiber sensor in the phase measurement," Sensors and Actuators B: Chemical, vol. 131, no. 2, pp. 1120-1124, 2008.

[130] M. Erdmanis, D. Viegas, M. Hautakorpi, S. Novotny, J. Santos, and H. Ludvigsen, "Comprehensive numerical analysis of a surface-plasmon-resonance sensor based on an H-shaped optical fiber," Optics Express, vol. 19, no. 15, pp. 13980-13988, 2011.

[131] R. Slavik, J. Homola, and J. Ctyroky, "Miniaturization of fiber optic surface Plasmon resonance sensor," Sensors and Actuators B: Chemical, vol. 51, no. 1-3, pp. 311-315, 1998.

[132] W. J. H. Bender, R. E. Dessy, M. S. Miller, and R. O. Claus, "Feasibility of a chemical microsensor based on surface plasmon resonance on fiber optics modified by multilayer vapor deposition," Analytical Chemistry, vol. 66, no. 7, pp. 963-970, 1994.

[133] M. Piliarik, J. Homola, Z. Manikova, and J. Ctyroky, "Surface plasmon resonance sensor based on a single-mode polarization-maintaining optical fiber," Sensors and Actuators B: Chemical, vol. 90, no. 1-3, pp. 236-242, 2003.

[134] M. H. Chiu, C. H. Shih, and M. H. Chi, "Optimum sensitivity of single-mode D-type optical fiber sensor in the intensity measurement," Sensors and Actuators B: Chemical, vol. 123, no. 2, pp. 1120-1124, 2007.

[135] R. Slavik, J. Homola, J. Ctyroky, and E. Brynda, "Novel spectral fiber optic sensor based on surface plasmon resonance," Sensors and Actuators B: Chemical, vol. 74, no. 1-3, pp. 106-111, 2001.

[136] Y. J. He, Y. L. Lo, and J. F. Huang, "Optical-fiber surface-plasmon-resonance sensor employing long-period fiber gratings in multiplexing," Journal of the Optical Society of America B, vol. 23, no. 5, pp. 801-811, 2006.

[137] J. L. Tang, S. F. Cheng, W. T. Hsu, T. Y. Chiang, and L. K. Chau, "Fiber-optic biochemical sensing with a colloidal gold-modified long period fiber grating," Sensors and Actuators B: Chemical, vol. 119, no. 1, pp. 105-109, 2006.

[138] G. Nemova and R. Kashyap, "Fiber Bragg grating assisted surface plasmon polariton sensor," Optics Letters, vol. 31, no. 14, pp. 2118-2120, 2006.

[139] G. Nemova and R. Kashyap, "Modeling of plasmon-polariton refractive-index hollow core fiber sensors assisted by a fiber Bragg grating," Journal of Lightwave Technology, vol. 24, no. 10, pp. 3789-3796, 2006.

[140] T. Allsop, R. Neal, S. Rehman, D. J. Webb, D. Mapps, and I. Bennion, "Characterization of infrared surface plasmon resonances generated from a fiber-optical sensor utilizing tilted Bragg gratings," 
Journal of the Optical Society of America B, vol. 25, no. 4, pp. 481-490, 2008.

[141] W. Ding, S. R. Andrews, T. A. Birks, and S. A. Maier, "Modal coupling in fiber tapers decorated with metallic surface gratings," Optics Letters, vol. 31, no. 17, pp. 2556-2558, 2006.

[142] B. Gauvreau, A. Hassani, M. F. Fehri, A. Kabashin, and M. Skorobogatiy, "Photonic bandgap fiber-based surface plasmon resonance sensors," Optics Express, vol. 15, no. 18, pp. 11413-11426, 2007.

[143] M. Hautakorpi, M. Mattinen, and H. Ludvigsen, "Surface-plasmon-resonance sensor based on three-hole microstructured optical fiber," Optics Express, vol. 16, no. 12, pp. 8427-8432, 2008.

[144] A. Hassani and M. Skorobogatiy, "Design of the microstructured optical fiber-based surface plasmon resonance sensors with enhanced microfluidics," Optics Express, vol. 14, no. 24, pp. 11616-11621, 2006.

[145] A. Hassani, B. Gauvreau, M. F. Fehri, A. Kabashin, and M. Skorobogatiy, "Photonic crystal fiber and waveguide-based surface plasmon resonance sensors for application in the visible and near-IR," Electromagnetics, vol. 28, no. 3, pp. 198-213, 2008.

[146] S. G. Johnson, S. H. Fan, P. R. Villeneuve, J. D. Joannopoulos, and L. A. Kolodziejski, "Guided modes in photonic crystal slabs," Physical Review B, vol. 60, no. 8, pp. 5751-5758, 1999.

[147] S. Fan and J. D. Joannopoulos, "Analysis of guided resonances in photonic crystal slabs," Physical Review B, vol. 65, no. 23, pp. 235112-1-235112-8, 2002.

[148] A. Ricciardi, I. Gallina, S. Campopiano, G. Castaldi, M. Pisco, V. Galdi, et al., "Guided resonances in photonic quasicrystals," Optics Express, vol. 17, no. 8, pp. 6335-6346, 2009.

[149] M. Pisco, A. Ricciardi, I. Gallina, G. Castaldi, S. Campopiano, A. Cutolo, et al., "Tuning efficiency and sensitivity of guided resonances in photonic crystals and quasi-crystals: a comparative study," Optics Express, vol. 18, no. 16, pp. 17280-17293, 2010.

[150] A. Ricciardi, M. Pisco, I. Gallina, S. Campopiano, V. Galdi, L. O'Faolain, et al., "Experimental evidence of guided-resonances in photonic crystals with aperiodically ordered supercells," Optics Letters, vol. 35, no. 23, pp. 3946-3948, 2010.

[151] A. Ricciardi, M. Pisco, A. Cutolo, A. Cusano, L. O'Faolain, T. F. Krauss, et al., "Evidence of guided resonances in photonic quasicrystal slabs," Physical Review B, vol. 84, no. 8, pp. 085135-1-085135-4, 2011.

[152] X. Yu, L. Shi, D. Han, J. Zi, and P. V. Braun, "High quality factor metallodielectric hybrid plasmonic-photonic crystals," Advanced Functional
Materials, vol. 20, no. 12, pp. 1910-1916, 2010.

[153] S. D. Hart, G. R. Maskaly, B. Temelkuran, P. Prideaux, J. D. Joannopoulos, and Y. Fink, "External reflection from omnidirectional dielectric mirror fibers," Science, vol. 296, no. 5567, pp. 510-513, 2002.

[154] M. Bayindir, A. F. Abouraddy, F. Sorin, J. D. Joannopoulos, and Y. Fink, "Fiber photodetectors codrawn from conducting, semiconducting and insulating materials," Optics and Photonics News, vol. 15, no. 12, pp. 14-24, 2004.

[155] M. Bayindir, F. Sorin, S. Hart, O. Shapira, J. D. Joannopoulos, and Y. Fink, "Metal-insulatorsemiconductor optoelectronic fibre" Nature, vol. 431, no. 7010, pp. 826-829, 2004.

[156] K. Kuriki, O. Shapira, S. D. Hart, G. Benoit, Y. Kuriki, J. Viens, et al., "Hollow multilayer photonic bandgap fibers for NIR applications," Optics Express, vol. 12, no. 8, pp. 1510-1517, 2004.

[157] M. Bayindir, O. Shapira, D. Saygin-Hinczewski, J. Viens, A. F. Abouraddy, J. D. Joannopoulos, et al., "Integrated Fibers for self monitored optical transport," Nature Materials, vol. 4, no. 11, pp. 820-824, 2005.

[158] M. Bayindir, A. F. Abouraddy, J. Arnold, J. D. Joannopoulos, and Y. Fink, "Thermal-sensing fiber devices by multimaterial codrawing," Advanced Materials, vol. 18, no. 7, pp. 845-849, 2006.

[159] M. Bayindir, A. F. Abouraddy, O. Shapira, J. Viens, D. Saygin-Hinczewski, F. Sorin, et al., "Kilometer-long ordered nanophotonic devices by preform-to-fiber fabrication," IEEE Journal of Selected Topics in Quantum Electronics, vol. 12, no. 6, pp. 1202-1023, 2006.

[160] S. Egusa, Z. Wang, N. Chocat, Z. M. Ruff, A. M. Stolyarov, D. Shemuly, et al., "Multimaterial piezoelectric fibres," Nature Materials, vol. 9, no. 8, pp. 643-648, 2010.

[161] F. Sorin and Y. Fink, "Multimaterial fiber sensors," in Proc. SPIE, vol. 7653, pp. 765305-1-765305-9, 2010.

[162] E. J. Smythe, M. D. Dickey, J. Bao, G. M. Whitesides, and F. Capasso, "Optical antenna arrays on a fiber facet for in situ surface-enhanced Raman scattering detection," Nano Letters, vol. 9, no. 3, pp. 1132-1138, 2009.

[163] I. W. Jung, B. Park, J. Provine, R. T. Howe, and O. Solgaard, "Highly sensitive monolithic silicon photonic crystal fiber tip sensor for simultaneous measurement of refractive index and temperature," Journal of Lightwave Technology, vol. 29, no. 9, pp. 1367-1374, 2011.

[164] S. Scheerlinck, P. Dubruel, P. Bienstman, E. Schacht, D. Van Thourhout, and R. Baets "Metal grating 
patterning on fiber facets by UV-based nano imprint and transfer lithography using optical alignment," Journal of Lightwave Technology, vol. 27, no. 10, pp. 1415-1420, 2009.

[165] D. Iannuzzi, K. Heeck, M. Slaman, S. de Man, J. H. Rector, H. Schreuders, et al., "Fibre-top cantilevers: design, fabrication and applications," Measurement Science and Technology, vol. 18, no. 10, pp. 3247-3252, 2007.

[166] A. A. Said, M. Dugan, S. de Man, and D. Iannuzzi, "Carving fiber-top cantilevers with femtosecond laser micromachining," Journal of Micromechanics and Microengineering, vol. 18, no. 3, pp. 35005-35008, 2008.

[167] G. Gruca, S. de Man, M. Slaman, J. H. Rector, and D. Iannuzzi, "Ferrule-top micromachined devices: design, fabrication, performance," Measurement Science and Technology, vol. 21, no. 9, pp. 94033-94038, 2010.

[168] D. Iannuzzi, S. Deladi, M. Slaman, J. H. Rector, H. Schreuders, and M. C. Elwenspoek, "A fiber-top cantilever for hydrogen detection," Sensors and Actuators B: Chemical, vol. 121, no. 2, pp. 706-708, 2006.
[169] C. J. Alberts, S. De Man, J. W. Berenschot, V. J. Gadgil, M. C. Elwenspoek, and D. Iannuzzi, "Fiber-top refractometer," Measurement Science and Technology, vol. 20, no. 3, pp. 034005-1-034005-5, 2009.

[170] D. Iannuzzi, S. Deladi, J. W. Berenschot, S. De Man, K. Heeck, and M. C. Elwenspoek, "Fiber-top atomic force microscope," Review of Scientific Instruments, vol. 77, no. 10, pp. 106105-1-106105-3, 2006.

[171] A. Dhawan, M. D. Gerhold, and J. F. Muth, "Plasmonic structures based on subwavelength apertures for chemical and biological sensing applications," IEEE Sensors Journal, vol. 8, no. 6, pp. 942-950, 2008.

[172] Y. Lin, Y. Zou, and R. G. Lindquist, "A reflection-based localized surface plasmon resonance fiber-optic probe for biochemical sensing," Biomedical Optics Express, vol. 2, no. 3, pp. 478-484, 2011.

[173] D. Rosenblatt, A. Sharon, and A. A. Friesem, "Resonant grating waveguide structures," IEEE Journal Quantum Electronics, vol. 33, no. 11, pp. 2058-2059, 1997. 